\title{
Flexible utilization of spatial- and motor-based codes for the storage of visuo- spatial information
}

Margaret Henderson ${ }^{1,2,3}$, Rosanne L. Rademaker ${ }^{4,5}$, John T. Serences ${ }^{1,4,6}$

${ }^{1}$ Neurosciences Graduate Program, University of California, San Diego, La Jolla, California 92093-1090 USA
2Department of Machine Learning, Carnegie Mellon University, Pittsburgh, Pennsylvania 15213 USA
${ }^{3}$ Neuroscience Institute, Carnegie Mellon University, Pittsburgh, Pennsylvania 15213 USA
${ }^{4}$ Department of Psychology, University of California, San Diego, La Jolla, California 92093-1090 USA
${ }^{5}$ Ernst Strüngmann Institute (ESI) for Neuroscience in Cooperation with Max Planck Society, Frankfurt, Germany
${ }^{6}$ Kavli Foundation for the Brain and Mind, University of California, San Diego, La Jolla, California 92093 USA

Author Contributions:

All authors conceived the experiments and wrote/edited the manuscript. $\mathrm{MH}$ and RR implemented the experiments, $\mathrm{MH}$ collected and analyzed the data, $\mathrm{MH}$ wrote the first draft of the manuscript.

\section{Correspondence:}

Margaret Henderson

Machine Learning Department, School of Computer Science

Carnegie Mellon University

5000 Forbes Avenue, Pittsburgh, PA 15213

mmhender@cmu.edu

Acknowledgements: Funded by NEI R01-EY025872 to JS, NIMH Training Grant in Cognitive Neuroscience (T32-MH020002) to MH, and a European Union's Horizon 2020 research and innovation program under the Marie Sklodowska-Curie Grant Agreement No 743941 to RR. We thank Kirsten Adam, Timothy Sheehan, and Sunyoung Park for helpful discussions and data collection.

Authors report no conflict of interest. 


\begin{abstract}
(214 words)
Working memory (WM) provides flexible storage of information in service of upcoming behavioral goals. Some models propose specific fixed loci and mechanisms for the storage of visual information in WM, such as sustained spiking in parietal and prefrontal cortex during the maintenance of features. An alternative view is that information can be remembered in a flexible format that best suits current behavioral goals. For example, remembered visual information might be stored in sensory areas for easier comparison to future sensory inputs (i.e. a retrospective code) or might be remapped into a more abstract, output-oriented format and stored in motor areas (i.e. a prospective code). Here, we tested this hypothesis using a visual-spatial working memory task where the required behavioral response was either known or unknown during the memory delay period. Using fMRI and multivariate decoding, we found that there was less information about remembered spatial positions in early visual and parietal regions when the required response was known versus unknown. Further, a representation of the planned motor action emerged in primary somatosensory, primary motor, and premotor cortex on the same trials where spatial information was reduced in early visual cortex. These results suggest that the neural networks supporting WM can be strategically reconfigured depending on the specific behavioral requirements of canonical visual WM paradigms.
\end{abstract}




\section{Introduction}

Working memory (WM) is thought to provide a flexible mental workspace which allows organisms to hold information in mind about past experiences and use it to guide future behavior (Baddeley \& Hitch, 1974). This system supports a wide range of cognitive tasks, each with its own specific demands and processing constraints. Due to these varied demands, it is likely that the neural mechanisms of WM are not universal across all tasks, but adaptively adjust to the requirements of the current situation. Among the many possible differences in task requirements, one key factor is the degree to which a task encourages a "retrospective" versus a "prospective" coding format. For instance, in tasks that require memory for fine visual details, such as searching for a specific kind of bird based on a picture in a guidebook, the best strategy might be to represent information in a format resembling past visual inputs (a "retrospective" code). Other tasks, such as remembering a series of directions for driving to your friend's house, permit the use of multiple strategies. This driving task could be achieved using a retrospective strategy, such as maintaining a visuo-spatial representation of a street map. However, a better approach might be to re-map the visual information into another format, such as a series of motor plans for upcoming actions (a "prospective" code). Such flexible remapping of information from retrospective to prospective codes could serve to reduce the dimensionality of representations when the correspondence between a remembered stimulus and a required action is known in advance. Thus, even tasks that are often thought to share a core component (e.g., memory in a visual format) might be accomplished via very different strategies and neural codes.

The paradigms used in human neuroimaging experiments typically sit at the retrospective end of this continuum. In most fMRI studies of visual WM, participants are required to remember precise values of continuously varying features, and to report these remembered features using responses that cannot be pre-planned during the delay period (Albers, Kok, Toni, Dijkerman, \& De Lange, 2013; Christophel, Hebart, \& Haynes, 2012; Ester, Serences, \& Awh, 2009; Harrison \& Tong, 2009; Lorenc, Sreenivasan, Nee, Vandenbroucke, \& D'Esposito, 2018; Rademaker, Chunharas, \& Serences, 2019; Serences, Ester, Vogel, \& Awh, 2009; Xing, Ledgeway, McGraw, \& Schluppeck, 2013). Such tasks may encourage top-down recruitment of the same early sensory areas that support high-precision perceptual representations (Awh \& Jonides, 2001; Gazzaley \& Nobre, 2012; Pasternak \& Greenlee, 2005; Serences, 2016; Sreenivasan, Curtis, \& D'Esposito, 2014). Consistent with this idea of sensory recruitment, most studies find that patterns of voxel activation measured in visual cortex encode information about specific feature values held in memory, supporting the role of visual cortex in maintaining detailed visual representations during WM.

However, different neural loci are often implicated using visual WM tasks that enable prospective or motor-oriented codes. One example is a memory-guided saccade task employed to study spatial WM using nonhuman primate (NHP) electrophysiological approaches. In these tasks, the position of a briefly presented cue is remembered, but that position is also predictive of the saccade that must be made at the end of the trial. Thus, the animal could solve the task by re-mapping information from a retrospective spatial code into a prospective motor code. Single unit recordings made during these tasks suggest an important role for the prefrontal cortex (PFC) in maintaining remembered information across brief delay periods (Funahashi, Bruce, \& Goldman-Rakic, 1989; Fuster \& Alexander, 1971; Goldman-Rakic, 1995). Consistent with these findings, others have suggested that prospective WM in general may rely more heavily on areas involved in planning and motor production, and less on early sensory cortex (Boettcher, Gresch, Nobre, \& Van Ede, 2021; Curtis, Rao, \& D’Esposito, 2004; Myers, Stokes, \& Nobre, 2017).

Based on the work discussed above, visual WM may be implemented quite flexibly, as opposed to having a singular neural locus or mechanism. Consistent with this idea, there are indications that even 
information related to the same stimulus might be stored at several different loci and in different formats (Iamshchinina, Christophel, Gayet, \& Rademaker, 2021; Lee, Kravitz, \& Baker, 2013; Rademaker et al., 2019; Serences, 2016). At the same time, few experiments have directly tested whether representations of memorized features in early visual cortex are subject to task modulation. Indeed, an alternative theory of WM storage suggests that memory-related signals in early sensory areas are epiphenomenal, in which case task goals are not expected to affect the strength of representations in this part of the brain (Leavitt, Mendoza-Halliday, \& Martinez-Trujillo, 2017; Xu, 2020). Thus, our understanding of how behavioral requirements influence the memory networks and the codes that route information between early sensory and other brain regions is incomplete.

Here we study visuo-spatial WM to determine if the neural mechanisms supporting information storage are flexible in the face of changing behavioral requirements, or if, alternatively, the same mechanisms are recruited irrespective of the ability to employ different strategies. Participants performed a visual-spatial WM task in which the required behavioral response on each trial was either not known until the end of the memory delay ("uninformative" condition), or known in advance of the memory delay ("informative" condition; Figure 1A). This manipulation was intended to promote the use of retrospective and prospective memory codes, respectively. We then compared representations of retrospective and prospective mnemonic information in early visual, parietal, and sensorimotor cortical regions.

To preview, we found that information about remembered spatial positions in retinotopic visual cortex decreased when the required response was known in advance, in accordance with a decreased reliance on visual cortex for information storage when prospective coding could be utilized. Furthermore, this decrease was accompanied by the emergence of a prospective memory representation in somatosensory, motor, and premotor cortex. Cross-generalization of decoding from independent modeltraining tasks further supports a shift from 'sensory-like' to 'motor-like' when comparing the two task conditions. These results demonstrate that the neural networks supporting WM - even in the context of a single paradigm often used to study visuo-spatial WM - can be strategically reconfigured depending on task requirements.

\section{$\underline{\text { Results }}$}

While in the MRI scanner, participants performed a spatial working memory task in which they were required to remember the spatial position of a briefly presented target dot across a 16-second delay period. The small white target dot could appear anywhere along an invisible circle with radius of $7^{\circ}$. After the delay, participants reported the dot's position by comparing their memory to a response probe $-\mathrm{a}$ disk with two halves (light and dark gray). They used their left or right index finger to indicate on which of the two halves of the disk the target dot had been presented (Figure 1A). We manipulated participants' ability to pre-plan their motor action by presenting a "preview" disk at the beginning of the delay period. The preview disk was preceded by a cue indicating whether the trial was part of the "informative" or "uninformative" condition. On informative trials, the preview disk matched the response disk, allowing participants to anticipate their button press at the end of the delay. On uninformative trials, the preview disk orientation was random with respect to that of the response disk, requiring that participants maintain a precise representation of the target dot position. Informative and uninformative trials were randomly intermixed throughout each run.

Task performance was overall better on trials when participants could plan their motor action in advance compared to trials when they could not (Figure 1C). Participants were significantly faster (informative mean \pm SEM: $0.57 \pm 0.03 \mathrm{sec}$; uninformative: $1.08 \pm 0.06 \mathrm{sec} ; \mathrm{t}_{(5)}=-9.618 ; \mathrm{p}<0.001$ ) and more 
bioRxiv preprint doi: https://doi.org/10.1101/2021.07.08.451663; this version posted July 9, 2021. The copyright holder for this preprint (which was not certified by peer review) is the author/funder. All rights reserved. No reuse allowed without permission.

accurate in the informative condition (informative mean \pm SEM: $93.92 \pm 2.12 \%$; uninformative: $89.83 \pm$ $1.12 \% ; t_{(5)}=3.463 ; p=0.018$ ). These behavioral benefits, particularly the response-time difference, suggest that participants used the preview disk to pre-plan their motor action in the informative condition. 
A

$A$

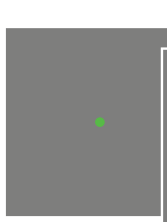

target

$0.75 \mathrm{~s}$

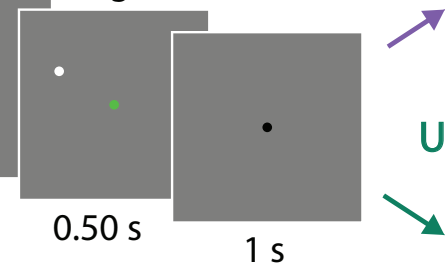

Informative

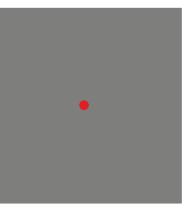

Uninformative

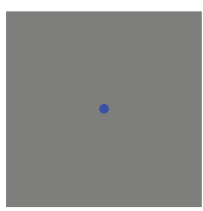

$2 \mathrm{~s}$
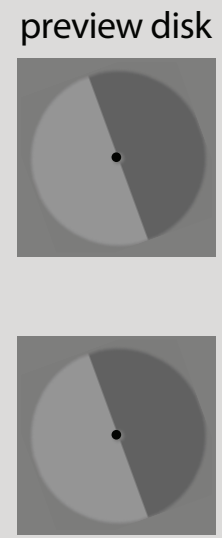

$1 \mathrm{~s}$

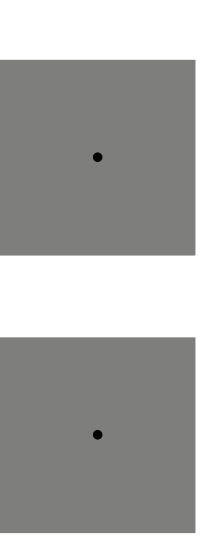

$12 \mathrm{~s}$ response disk
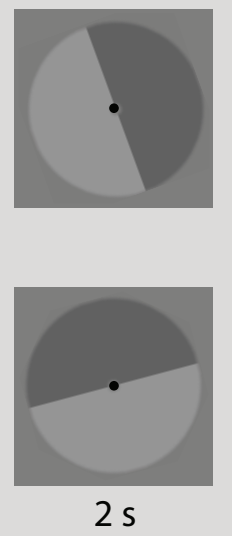

Informative
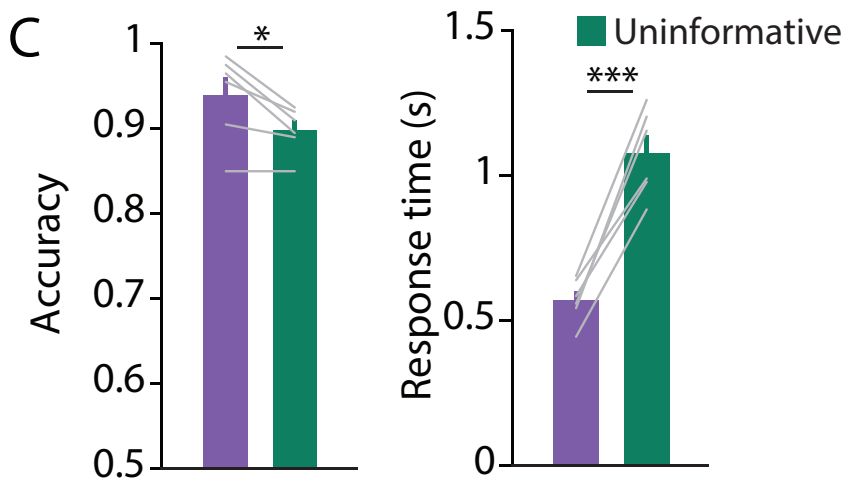

D

B

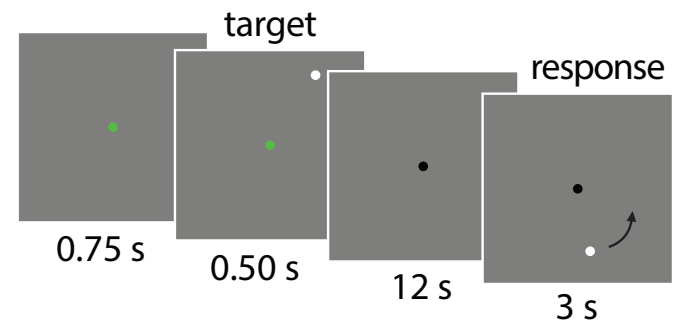

V1

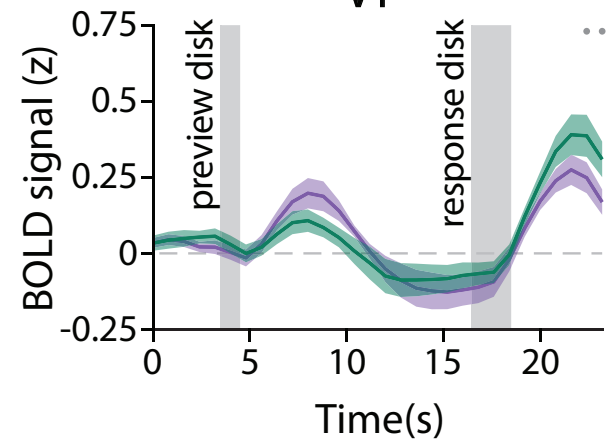

IPSO

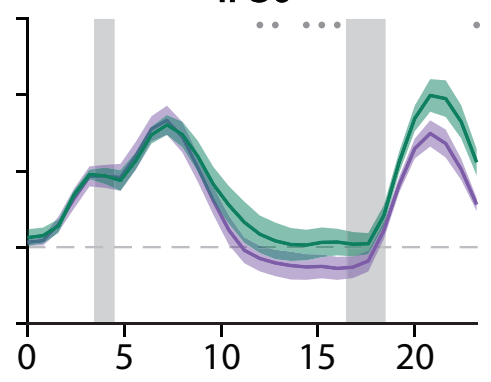

M1

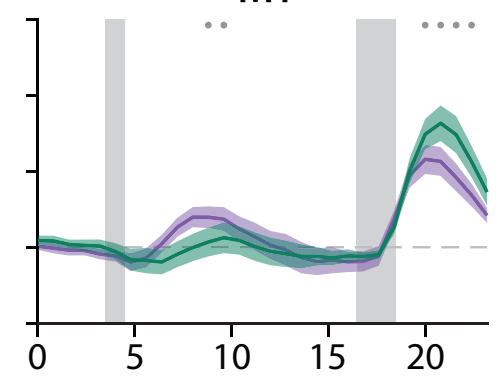

Figure 1. Task design, behavioral results, and univariate results. (A) During each trial of the main working memory task, participants remembered the spatial position of a target dot presented at a random angular position $\left(7^{\circ}\right.$ eccentricity from fixation). After a $16 \mathrm{sec}$ delay, participants made a binary response to indicate which half of a "response" disk the target dot position had been presented on. At the beginning of the delay, a "preview" disk was shown that either exactly matched the response disk (top row; "informative") or had a random orientation relative to the response disk (bottom row; "uninformative"). In the example trial depicted, a participant in the informative condition would have pressed the button corresponding to the lighter gray side of the disk. By contrast, in the uninformative condition a participant would have pressed the button corresponding to the dark gray side, as only the final response disk was relevant to their response (see Methods, Task: Main Working Memory for more details). (B) In a separate spatial working memory mapping task, participants remembered a target dot position for 12 seconds and responded by moving a probe dot around an invisible circle to match the remembered position (see Methods, Task: Spatial Working Memory Mapping). This mapping task was used to generate an independent data 
set to train decoding models (see Methods, Analysis: Spatial Position Decoding). (C) Average accuracy (left) and behavioral response time (right) in the informative and uninformative conditions (individual participants are shown with gray lines). Error bars represent \pm 1 SEM across participants. Significance of condition differences was computed using paired t-tests (see main text for exact $p$-values). (D) Univariate hemodynamic response functions (HRFs) in three representative regions of interest (ROIs) from early visual cortex (V1), parietal cortex (IPSO), and motor cortex (M1) during the informative (purple) and uninformative (green) conditions. Timepoint zero indicates the onset of the memory target. Shaded gray rectangles indicate the time periods when the "preview" disk was onscreen (3.5$4.5 \mathrm{sec})$ and when the response disk was onscreen $(16.5-18.5 \mathrm{sec})$. Shaded error bars represent \pm 1 SEM across participants. Gray dots indicate timepoints showing a significant condition difference, evaluated using a Wilcoxon signed-rank test with permutation, all p-values $<0.05$; see Methods, Analysis: Univariate for details). This plot shows three representative ROIs, see Supplementary Figure 1 for data from all ROIs.

Next, we examined the average fMRI responses in both visual and sensorimotor cortical areas, which were each independently localized (see Methods, Identifying Regions of Interest). Our visual regions of interest (ROIs) were retinotopically-defined areas in occipital and parietal cortex, and our sensorimotor ROIs were action-related regions of primary somatosensory cortex (S1), primary motor cortex (M1), and premotor cortex (PMc). We used linear deconvolution to calculate the average hemodynamic response function (HRF) for voxels in each ROI during each task condition (see Methods, Analysis: Univariate; Dale, 1999; Dale \& Buckner, 1997).

As expected, the BOLD signal in all retinotopic ROls increased following visual stimulation (Figure 1D; Supplementary Figure 1). We also replicated the typical finding that occipital retinotopic areas V1-V4 did not show sustained BOLD activation during the memory delay period in either task condition (Offen, Schluppeck, \& Heeger, 2009; Riggall \& Postle, 2012; Serences et al., 2009). Also as expected, mean BOLD responses in parietal areas IPSO-3 showed elevated late delay period activation in the uninformative condition relative to the informative condition. This pattern is consistent with the use of a retrospective spatial code in the uninformative condition and a prospective motor code in the informative condition (Curtis \& D'Esposito, 2003; D’Esposito, 2007; Ester, Sprague, \& Serences, 2015; Riggall \& Postle, 2012). Sensorimotor areas S1, M1, and PMc showed a condition difference in the opposite direction, with higher mean BOLD responses in the informative compared to the uninformative condition at several timepoints early in the delay period, consistent with an increased reliance on a prospective memory code (Calderon, Van Opstal, Peigneux, Verguts, \& Gevers, 2018; Donner, Siegel, Fries, \& Engel, 2009).

The univariate results described above are consistent with the idea of an information "handoff" between cortical regions as a function of retrospective and prospective task strategies. This brings up the important question of how such a task-related shift is reflected in population-level representations across the brain, and whether it coincides with a shift from a sensory-like retrospective memory code to a more motor-like prospective memory code. To evaluate this question, we used a multivariate linear classifier to decode the angular spatial position of the remembered dot, based on multi-voxel activation patterns measured in each ROI during the delay period (Figure 2). By assessing spatial decoding accuracy in each $\mathrm{ROI}$, we could assess the extent to which the underlying neural code reflected a retrospective visual representation of memorized spatial position.

To facilitate an independent comparison of decoding accuracy between the informative and the uninformative task conditions in the main working memory task, we used data from an independent spatial working memory task to train the classifier (Sprague, Boynton, \& Serences, 2019) (Figure 1B; see Methods, Task: Spatial Working Memory Mapping). Before performing the decoding analysis, we subtracted from each single-trial activation pattern the mean across voxels on the same trial. This was done to ensure that any condition-specific changes in the mean BOLD responses did not contribute to 
bioRxiv preprint doi: https://doi.org/10.1101/2021.07.08.451663; this version posted July 9, 2021. The copyright holder for this preprint (which was not certified by peer review) is the author/funder. All rights reserved. No reuse allowed without permission.

differences in classification accuracy (see Methods: Analysis: Spatial Position Decoding). We then sorted the continuous angular positions into 8 non-overlapping bins and used a decoding scheme with four binary classifiers, where each binary classifier was independently trained to discriminate between spatial positions that fell into bins separated by $180^{\circ}$ (see Figure $2 \mathrm{~A}$ and Rademaker et al., 2019). The final decoding accuracy for each task condition reflects the average decoding accuracy across all four of these binary classifiers, where chance is 50\% (see Methods, Analysis: Spatial Position Decoding). 


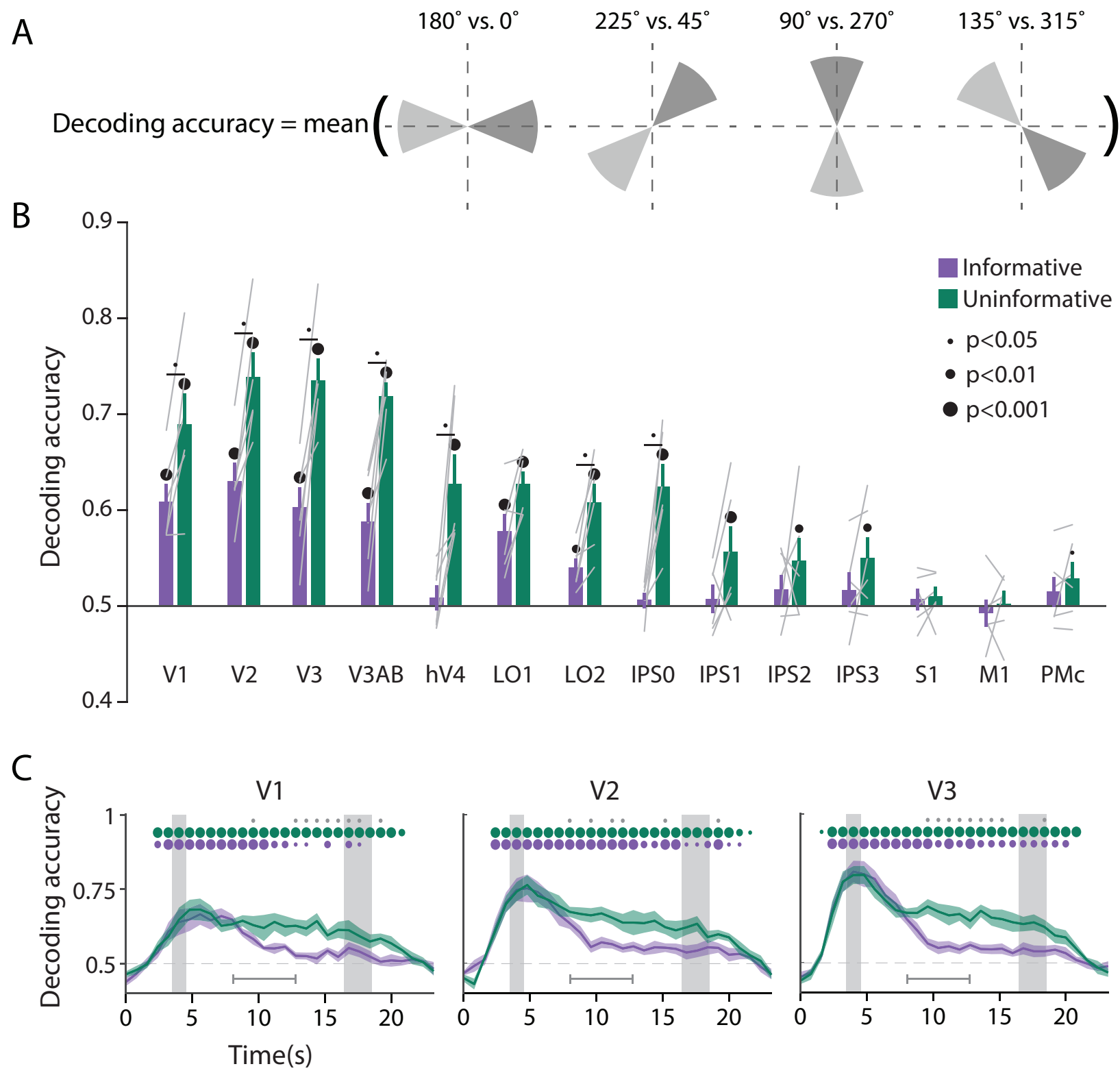

Figure 2. Visual and parietal areas represent spatial position more strongly during trials that require retrospective spatial memory than during trials that allow prospective motor memory. (A) Schematic of the decoding procedure. Continuous values of angular position were divided into 8 discrete bins, and 4 binary decoders were trained to discriminate between patterns corresponding to bins $180^{\circ}$ apart. The final decoding accuracy was the average accuracy over these 4 decoders. (B) Decoding accuracy for each ROI and task condition. The spatial decoder was always trained on data from the delay period of an independent spatial working memory mapping task (Fig 1B), and tested on data from the delay period of the main working memory task (averaged within a window 8-12.8 sec from start of trial; see Methods, Analysis: Spatial Position Decoding for more details). Error bars reflect \pm 1 SEM across participants, and light gray lines indicate individual participants. Dots above bars and pairs of bars indicate the level of statistical significance within each condition, and between conditions, respectively (two-tailed p-values obtained using a Wilcoxon signed-rank test with permutation testing, see Methods, Analysis: Spatial Position Decoding). Dot sizes reflect significance level. (C) Spatial decoding accuracy over time in three example ROls. Timepoint zero indicates the target onset time. Shaded gray rectangles indicate the periods of time when the "preview" (3.5-4.5 $\mathrm{sec}$ ) and "response" (16.5-18.5 sec) disks were onscreen. Shaded error bars represent \pm 1 SEM across participants, colored dots indicate significance of decoding within each condition, and gray dots indicate significant condition 
differences, with dot sizes reflecting significance levels as in B. Gray brackets in $\mathbf{C}$ indicate the time range in which data were averaged to produce B (i.e. 8-12.8 sec). See Supplementary Figure 2 for time-resolved decoding in all visual and motor ROIs.

The results of this multivariate analysis demonstrate that across the uninformative and the informative conditions, spatial decoding accuracy was strongest in early visual areas V1, V2, V3, and V3AB, and became progressively weaker at more anterior regions of the visual hierarchy (Figure 2B). Spatial decoding accuracy was at chance in the three sensorimotor areas S1, M1, and PMc. Importantly, there was also a pronounced effect of task condition (Figure 2B, 2C). Decoding accuracy in most retinotopic areas was significantly higher for the uninformative condition, in which participants were forced to rely on a retrospective spatial memory code, compared to the informative condition, in which participants could maintain a prospective memory for the button press required at the end of the delay (two-way repeated measures ANOVA with ROI and task condition as factors: main effect of ROI: $F_{(13,65)}=24.548, p<0.001$; main effect of task condition: $F_{(1,5)}=35.537, p=0.001$; ROI $x$ condition interaction $F_{(13,65)}=5.757, p<0.001$; p-values obtained using permutation test; see Methods, Analysis: Spatial Position Decoding). Pairwise comparisons showed that spatial decoding accuracy was significantly higher in the uninformative compared to the informative condition in V1-V4, LO2, and IPSO. In later IPS subregions (IPS1-3), spatial decoding was above chance in the uninformative memory condition, but at chance in the informative condition, without a significant difference between conditions. Finally, time-resolved analyses revealed that this difference between the conditions was not present during and immediately after encoding of the target dot, when the trial condition was not yet known. Instead, condition differences emerged approximately 5-6 seconds after the presentation of the preview disk and persisted until the final response disk appeared (Figure 2C, Supplementary Figure 2). Importantly, the difference in spatial decoding accuracy between task conditions replicated when we trained and tested a decoder using data from within each condition of the main working memory task as opposed to training on the independent spatial working memory task. This indicates that the difference between task conditions was robust to a range of analysis choices (Supplementary Figure 3; see Methods, Analysis: Spatial Position Decoding).

In addition to demonstrating the robustness of our findings, the two separate analysis approaches described above allow us to distinguish between two potential explanations for the observed condition difference. While one possibility is that visual cortex activation patterns contain less overall information about spatial position in the informative relative to the uninformative condition, another possibility is that the format of the representations differs between the two task conditions (e.g. Lorenc, Vandenbroucke, Nee, de Lange, \& D'Esposito, 2020; Vaziri-Pashkam \& Xu, 2017). Specifically, compared to the informative condition, the retrospective format used in the uninformative condition may have been more similar to the format of the independent spatial working memory task (which also required a retrospective strategy because the starting position of the response dot was unknown until the end of the trial; see Figure 1B). This similarity could have led to higher cross-generalization of the decoder to the uninformative condition, even if the overall spatial information content was similar between the two conditions in the main working memory task. The fact that the condition difference persists when training and testing within condition rules this latter possibility out. Instead, our analyses support the interpretation that spatial working memory representations in visual cortex are adaptively enhanced in the uninformative condition (when a retrospective code is required) relative to the informative condition (where a prospective code can be used).

In addition to retrospective spatial position decoding, we investigated the format of the mnemonic code during the informative condition, in which participants could maintain a prospective representation 
of their upcoming action. Since motor responses were always made with the left or the right index finger, we trained a binary linear classifier to predict which finger was associated with the required button-press on each trial based on delay period signal in each ROI (Figure 3A, see Methods, Analysis: Action Decoding for detailed classification procedure). This analysis revealed above chance decoding of prospective actions in each of the three sensorimotor ROIs (S1, M1, and PMC) in the informative condition but not the uninformative condition. In contrast, all retinotopic visual ROIs showed chance level action decoding for both conditions (two-way repeated measures ANOVA with ROI and task condition as factors: main effect of ROI: $F_{(13,65)}=4.003, p<0.001$; main effect of condition $F_{(1,5)}=3.802, p=0.106$; ROI $x$ condition interaction: $\mathrm{F}_{(13,65)}=2.937, \mathrm{p}=0.001 ; \mathrm{p}$-values obtained using permutation test; see Methods, Analysis: Action Decoding). A time-resolved decoding analysis revealed that information about a participants' upcoming action began to emerge approximately 4 seconds after the onset of the preview disk stimulus, decreased slightly toward the end of the delay period, then rose steeply after the response disk onset when the participant actually executed a motor action (Figure 3B, Supplementary Figure 4). The increase in action decoding accuracy during the last part of the trial appeared sooner for the informative condition than the uninformative condition, in agreement with the speeding of behavioral response times in the informative condition (Figure 1C). 


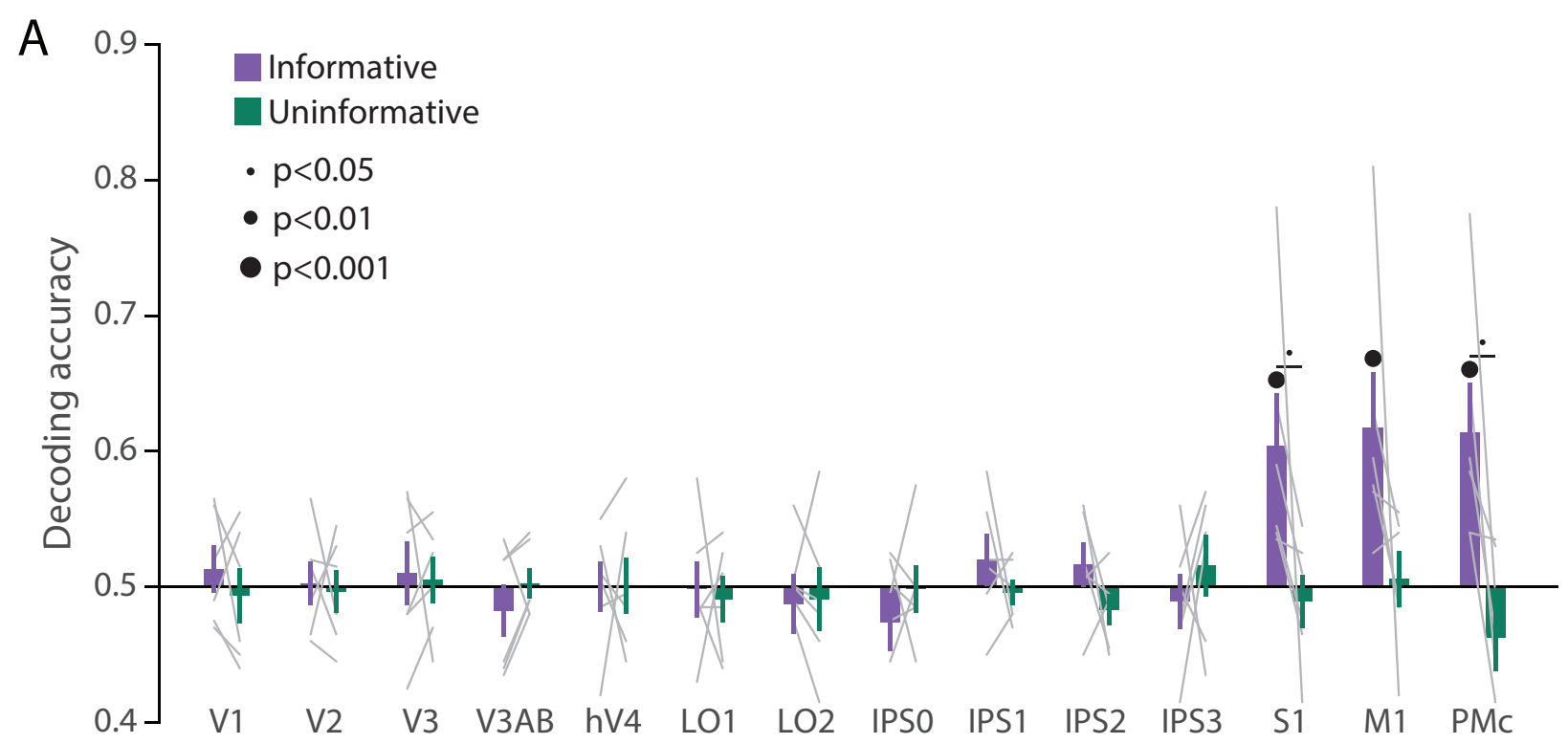

B
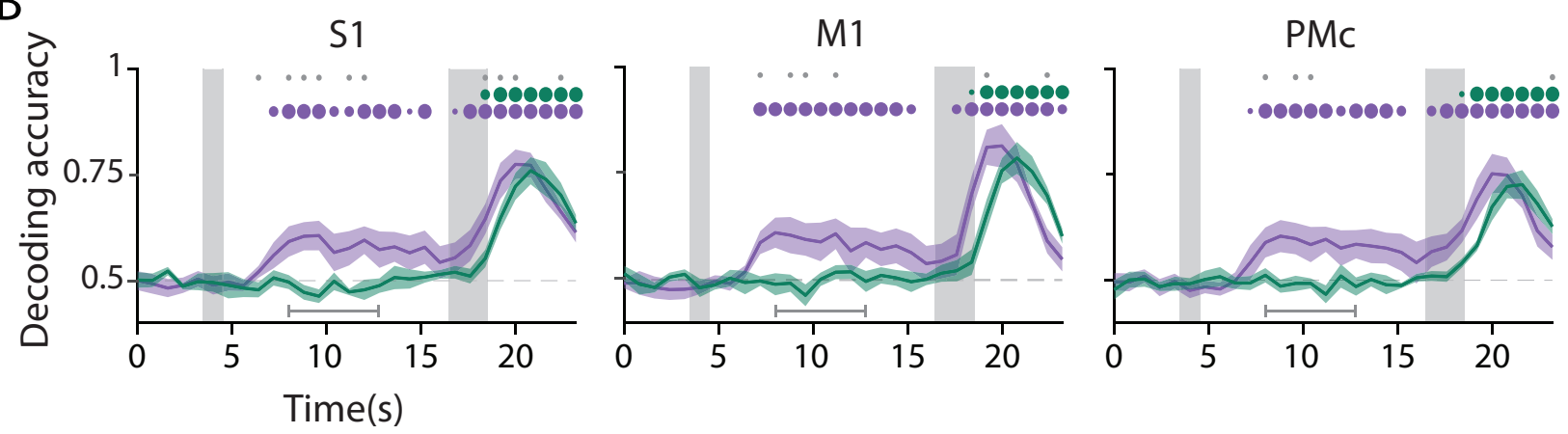

Figure 3. Prospective memory representations can be decoded from sensorimotor ROls during the delay period. (A) A linear decoder was trained to classify the finger (left or right index) associated with the correct motor action on each trial, using data measured during the delay period of trials in each task condition separately (averaged within a window 8-12.8 sec from start of trial; see Methods, Analysis: Action Decoding for more details). Error bars reflect \pm 1 SEM across participants, and light gray lines indicate individual participants. Dots above bars and pairs of bars indicate the statistical significance of decoding accuracy within each condition, and of condition differences, respectively (two-tailed p-values obtained using a Wilcoxon signed-rank test with permutation testing, see Methods, Analysis: Action Decoding). Dot sizes reflect significance level. (B) Action decoding accuracy over time in three example ROIs. Timepoint zero indicates the target onset time. Shaded gray rectangles indicate the periods during which the "preview" (3.5-4.5 sec) and "response" (16.5-18.5 sec) disks were onscreen. Shaded error bars represent \pm 1 SEM across participants. Colored dots indicate significance of decoding accuracy within each condition, and gray dots indicate significant condition differences, with dot sizes reflecting significance levels as in A. Gray brackets in B indicate the time range in which data were averaged to produce A (8-12.8 sec). For time-resolved decoding in all ROIs, see Supplementary Figure 4.

The above results support the hypothesis that the informative and uninformative conditions engaged different regions of cortex and different coding formats for information storage. To characterize this difference in coding format in a more concrete way, we next leveraged two additional independent model training tasks. First, we utilized a spatial localizer during which participants viewed high-contrast flickering checkerboards at various spatial positions (see Methods, Task: Spatial Localizer). With this dataset, we 
trained a classifier on sensory-driven spatial responses (using the same spatial position decoding method as described previously, see Figure $2 \mathrm{~A}$ ). We found that spatial position information generalized from this perceptual training data to both conditions of our main working memory task (Figure 4A). This result supports the idea that the coding format used during spatial working memory is sensory-like in nature, closely resembling the responses in visual cortex to spatially-localized perceptual input. Importantly, decoding performance in early visual and parietal areas was again higher for the uninformative than the informative condition. This implies that the uninformative condition of our task resulted in a stronger and more 'sensory-like' retrospective code. The second independent model training task was a sensorimotor cortex localizer where participants physically pressed buttons with their left and right index fingers (see Methods, Task: Sensorimotor Cortex Localizer). We trained a decoder on this task, labeling trials according to which finger was used to physically press the buttons on each trial. We then tested this decoder on delay period activation in our main WM task. This analysis revealed above-chance decoding accuracy of the participant's upcoming action in S1, M1, and PMc, in the informative condition only (Figure 4B). This result supports the notion that the representations used for prospective memory in the informative condition were stored in a more motor-like format which closely resembled signals measured during actual button press responses. 

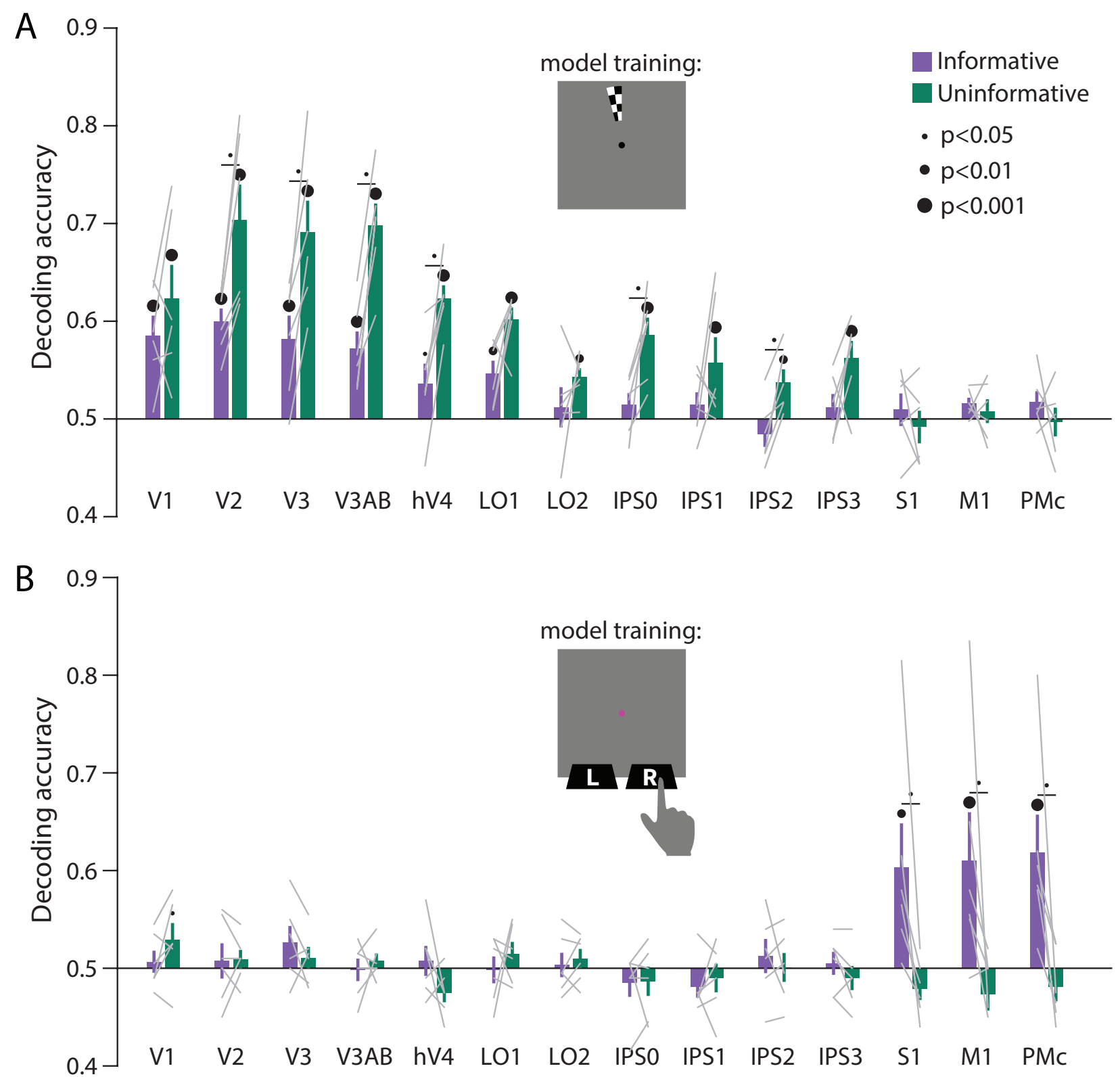

Figure 4. Retrospective and prospective memory representations generalize from signals associated with perceptual input and physical button-press responses, respectively. (A) Decoding accuracy of retrospective spatial memory position when training on data from an independent sensory localizer (see Methods, Task: Spatial Localizer \& Methods, Analysis: Spatial Position Decoding for details on this task and on decoding procedure). (B) Decoding accuracy of prospective memory codes (i.e. the finger associated with the correct motor action on each trial) when training on data from an independent button pressing task (see Methods, Task: Sensorimotor Cortex Localizer \& Methods, Analysis: Action Decoding for details on this task and on decoding procedure). In both panels, error bars reflect \pm 1 SEM across participants, and light gray lines indicate individual participants. Dots above bars and pairs of bars indicate the statistical significance of decoding accuracy within each condition, and of condition differences, respectively, both evaluated using non-parametric statistics. Dot sizes reflect significance level. Inserts show a cartoon of the localizer tasks used to train the decoder for these analyses. 


\section{Discussion}

Past studies of visual WM have led to competing theories of how information is stored in the brain. Some work has emphasized the recruitment of early sensory areas, while other work has emphasized parietal and frontal association areas. Nevertheless, a general consensus exists that WM is a flexible resource (Baddeley \& Hitch, 1974; Gazzaley \& Nobre, 2012; lamshchinina et al., 2021; Serences, 2016). Here we demonstrate, within a single spatial working memory paradigm, that task requirements are a critical determinant of how and where WM is implemented. These data provide a partial unifying explanation for divergent prior findings that implicate different regions in visual WM (Bettencourt \& Xu, 2015; Ester, Rademaker, \& Sprague, 2016; lamshchinina et al., 2021; Rademaker et al., 2019; Xu, 2018, 2020). More importantly, however, our data show that visual WM flexibly engages different cortical areas and coding formats, even in the context of a task that is commonly used to study a single underlying construct (i.e., visuo-spatial WM). These findings highlight the goal-oriented nature of WM: the brain's mechanisms for storage are not fully specified by the type of information being remembered, but instead depend on how the encoded information will be used to guide future behavior.

We used a spatial working memory task wherein participants could anticipate their behavioral response ahead of the delay interval on half of the trials (Figure $1 \mathrm{~A}$ ), encouraging a prospective memory representation. On the other half of trials, they were unable to anticipate their behavioral response, and had to rely on a retrospective memory representation. The decoding accuracy of a remembered position from activation patterns in visual cortex was lower when participants had the opportunity to prospectively plan their action, compared to when they were unable to plan (and thus had to rely exclusively on retrospective visual memory). This effect was consistent across a range of early visual and parietal ROls (Figure 2), supporting the idea that the recruitment of visual cortex for WM storage is task-dependent. Conversely, during the same trials where spatial representations became weaker in visual cortex, sensorimotor ROIs showed above-chance decoding of the participant's planned action during the delay period (Figure 3). We additionally show that the format of these two memory representations were markedly different: the spatial memory code generalized from an independent task in which stimuli at various spatial positions were actively viewed, whereas the action-related memory code generalized from an independent task in which participants made actual alternating button-press responses (Figure 4). Together, these results demonstrate that rather than having one fixed locus or mechanism, visual WM can be supported by different coding schemes that are adapted to current task requirements.

While we found widespread decoding of the contents of working memory, we generally did not find sustained increases in univariate BOLD responses during the delay (Figure 1D; Supplementary Figure 1). Specifically, visual areas V1-V4 and LO2 showed no sustained BOLD responses or differences in the mean BOLD signal between conditions during the delay period, despite these areas showing condition differences in spatial decoding accuracy. This adds to an existing body of research showing a dissociation between univariate and multivariate indices of WM storage (Emrich, Riggall, La Rocque, \& Postle, 2013; Ester et al., 2015; Harrison \& Tong, 2009; Riggall \& Postle, 2012; Serences et al., 2009). Further, this suggests that the observed differences in decoding performance cannot be explained by global changes in signal due to arousal or task difficulty, but are instead attributable to differences in the amount of information represented within population-level patterns of activation in visual cortex.

At the same time, in multiple subregions of IPS, we did observe higher univariate delay period activation for the uninformative condition relative to the informative condition. In contrast, the opposite was true in sensorimotor areas (Figure 1D; Supplementary Figure 1). This finding parallels previous work showing higher univariate delay period activation in IPS during a task where oculomotor responses were decoupled from spatial memory items (i.e. encouraging a retrospective strategy), whereas oculomotor 
areas showed higher activation when responses and memoranda were yoked (i.e. encouraging a prospective strategy; Curtis, Rao, \& D'Esposito, 2004). This past work suggested specialized networks for retrospective versus prospective memories. Our current demonstration of a shift from visual to sensorimotor codes further builds on this by showing how the neural systems underlying WM may be flexibly reconfigured, and memory representations flexibly reformatted, based on behavioral requirements.

We observed above-chance delay period decoding of participants' planned actions in S1, M1, and PMc. Each of these areas has previously been shown to exhibit preparatory motor activity in the context of tasks involving delayed reaching or finger pressing (Ariani, Pruszynski, \& Diedrichsen, 2020; Calderon et al., 2018; Cisek \& Kalaska, 2005; Donner et al., 2009). In our data, information regarding the prospective motor plan became detectable around 4 seconds after the onset of the preview disk - roughly the same time that spatial decoding accuracy in visual cortex dropped in the informative compared to the uninformative condition (Figure 2C, 3B). While suggestive, the temporal resolution of $\mathrm{fMRI}$ does not allow us to draw firm conclusions about the relative timing of each process. Nevertheless, these results are broadly consistent with a theory in which action selection unfolds in parallel to task-related processing of visual input (Cisek \& Kalaska, 2010; Donner et al., 2009; Klein-Flügge \& Bestmann, 2012; van Ede, Chekroud, Stokes, \& Nobre, 2019). Additionally, we found that information about prospective actions declined toward the end of the delay period, dropping below chance just before the response disk appeared (Figure 3B). This time course is consistent with a transient signal related to the initial formation of a prospective memory representation, and aligns with recent EEG findings in human motor cortex following a retro-cue in a visual WM task (Boettcher et al., 2021).

Beyond prospective and retrospective memory, other aspects of task requirements have been shown to influence the neural correlates of WM. For example, one experiment found that information about visual objects could be decoded from extrastriate visual areas on blocks of trials that required memory for visual details, but from PFC on blocks of trials where only the object's category had to be maintained (Lee et al., 2013). Other work has examined the effect of attentional priority on WM representational format, and has shown that high priority items tend to be represented more robustly than items that are not immediately relevant to behavior (Christophel et al., 2012; Lewis-Peacock, Drysdale, Oberauer, \& Postle, 2012; Lorenc et al., 2020; Rose et al., 2016; Sprague, Ester, \& Serences, 2016; Wolff, Jochim, Akyürek, \& Stokes, 2017) (but see also Barbosa, Soldevilla, \& Compte, 2021; lamshchinina et al., 2021). Prioritized WM representations can be further reconfigured so that they are optimized for future behavior, which may include the activation of circuits related to motor output (Myers et al., 2017; Nobre \& Stokes, 2019; Schneider, Barth, \& Wascher, 2017; Souza \& Oberauer, 2016; van Ede et al., 2019). These past studies of action preparation have typically used relatively coarse measures of visual information content such as decoding which of two items was currently selected. In contrast, here we measured the information about a continuous remembered feature value that was reflected in patterns of activation in visual cortex. We simultaneously showed a decrease in spatial information in visual cortex and an increase in action-related information in sensorimotor cortex when a prospective mnemonic format was encouraged. This finding solidifies the evidence showing that information may shift between qualitatively different kinds of codes as a function of behavioral requirements, as opposed to just waxing and waning over time within the same format/brain areas.

In contrast to human neuroimaging experiments, which often find evidence for sensory recruitment (i.e. WM decoding from early sensory cortices), research with non-human primates (NHPs) has generally found less evidence for maintenance of feature-specific codes in early sensory regions. The difference in results between human and primate studies may be partially accounted for by differences in measurement modality. For example, the BOLD response is driven in part by modulations of local field 
potentials (Boynton, 2011; Goense \& Logothetis, 2008; Logothetis, Pauls, Augath, Trinath, \& Oeltermann, 2001; Logothetis \& Wandell, 2004), which may contain information about remembered features, such as motion direction, even when single unit spike rates do not (Mendoza-Halliday, Torres, \& Martinez-Trujillo, 2014). Part of the discrepancy may also be related to differences in tasks used in human studies versus NHP studies. For example, some NHP studies use tasks that allow for prospective coding strategies, such as the traditional memory-guided saccade paradigm (Funahashi et al., 1989; Fuster \& Alexander, 1971; Goldman-Rakic, 1995). At the same time, other NHP experiments have dissociated sensory and actionrelated information (Funahashi, Chafee, \& Goldman-Rakic, 1993; Mendoza-Halliday et al., 2014; Miller, Erickson, \& Desimone, 1996; Panichello, DePasquale, Pillow, \& Buschman, 2019), suggesting that the difference between retrospective and prospective coding alone cannot account for differences across experiments. Another aspect of task design that may contribute to these differences is that NHP tasks often require animals to remember one of a small, discrete set of stimuli (e.g., Funahashi et al., 1993; Mendoza-Halliday et al., 2014; Miller et al., 1996). This may encourage a more discretized categorical representation rather than a highly detailed representation, which may rely less strongly on the finelytuned neural populations of early sensory areas (Lee et al., 2013). For example, some NHP electrophysiology studies have shown spiking activity in V1 that reflects the contents of WM using tasks that require precise spatial representations that may not be easily re-mapped into a non-sensory format (e.g., the curve-tracing task used by Van Kerkoerle and colleagues; Supèr, Spekreijse, \& Lamme, 2001; Van Kerkoerle, Self, \& Roelfsema, 2017).

Together, our findings suggest that the neural mechanisms that underlie WM are dynamic and can be flexibly adjusted, even in the context of a single paradigm typically used to study visuo-spatial WM. When our participants were given the possibility to switch from a purely visual to a prospective motor-based WM code, the amount of information regarding precise spatial position dropped in early visual and parietal cortex, while information about a prospective action became decodable from sensorimotor cortex. This experiment highlights how in a single paradigm, we can measure multiple dissociable mechanisms supporting WM. More broadly, these results open the door for future experiments to explore other task factors that may dynamically alter how WM is represented in the brain, and further push the boundaries of our knowledge about WM and cognitive flexibility.

\section{Materials and Methods}

Participants. 6 participants ( 2 male) between the ages of 20 and 34 were recruited from the UCSD community (mean age $27.2 \pm 2.7$ years). All had normal or corrected-to-normal vision. One additional participant (male) participated in an early pilot version of the study, but is not included here as they did not complete the final experiment. The study protocol was approved by the Institutional Review Board at UCSD, and all participants provided written informed consent. Each participant performed a behavioral training session lasting approximately 30 minutes, followed by 3 or 4 scanning sessions, each lasting approximately 2 hours. Participants were compensated at a rate of $\$ 10 /$ hour for behavioral training and $\$ 20 /$ hour for the scanning sessions. Participants were also given "bonus" money for correct performance on certain trials in the main behavioral task (see Task: Main Working Memory), up to a maximum of $\$ 40$ bonus.

Magnetic Resonance Imaging (MRI). All MRI scanning was performed on a General Electric (GE) Discovery MR750 3.0T research-dedicated scanner at the UC San Diego Keck Center for Functional Magnetic Resonance Imaging (CFMRI). Functional echo-planar imaging (EPI) data were acquired using a Nova Medical 32-channel head coil (NMSC075-32-3GE-MR750) and the Stanford Simultaneous Multi-Slice (SMS) EPI sequence (MUX EPI), with a multiband factor of 8 and 9 axial slices per band (total slices $=72 ; 2$ 
$\mathrm{mm}^{3}$ isotropic; $0 \mathrm{~mm}$ gap; matrix $=104 \times 104 ; \mathrm{FOV}=20.8 \mathrm{~cm}$; TR/TE $=800 / 35 \mathrm{~ms}$; flip angle $=52^{\circ}$; inplane acceleration $=1$ ). Image reconstruction and un-aliasing procedures were performed on servers hosted by Amazon Web Services, using reconstruction code from the Stanford Center for Neural Imaging. The initial 16 TRs collected at sequence onset served as reference images required for the transformation from kspace to image space. Two short (17 s) "topup" datasets were collected during each session, using forward and reverse phase-encoding directions. These images were used to estimate susceptibility-induced offresonance fields (Andersson, Skare, \& Ashburner, 2003) and to correct signal distortion in EPI sequences using FSL topup functionality (Jenkinson, Beckmann, Behrens, Woolrich, \& Smith, 2012).

In addition to the experimental scanning sessions, each participant participated in a separate retinotopic mapping session during which we also acquired a high-resolution anatomical scan. This anatomical T1 image was used for segmentation, flattening, and delineation of the retinotopic mapping data. For 4 out of the 6 participants, the anatomical scan was obtained using an Invivo 8-channel head coil with accelerated parallel imaging (GE ASSET on a FSPGR T1-weighted sequence; $1 \times 1 \times 1 \mathrm{~mm}^{3}$ voxel size; $8136 \mathrm{~ms}$ TR; $3172 \mathrm{~ms}$ TE; $8^{\circ}$ flip angle; 172 slices; $1 \mathrm{~mm}$ slice gap; 256x192 cm matrix size), and for the remaining 2 participants this scan was collected using the same 32-channel head coil used for functional scanning (anatomical scan parameters used with 32-channel coil were identical to those used with the 8channel coil). Anatomical scans collected with the 32-channel head coil were corrected for inhomogeneities in signal intensity using GE's "Phased array uniformity enhancement" (PURE) method.

Pre-Processing of MRI Data. All pre-processing of MRI data was performed using software tools developed and distributed by FreeSurfer and FSL (available at https://surfer.nmr.mgh.harvard.edu and http://www.fmrib.ox.ac.uk/fsl). First, we used the recon-all utility in the FreeSurfer analysis suite (Dale, Fischl, \& Sereno, 1999) to perform cortical surface gray-white matter volumetric segmentation of anatomical T1 scans. The segmented T1 data were used to define cortical meshes on which we specified retinotopic ROIs used for subsequent analyses (see Identifying ROIs). T1 data were also used to align multisession functional data into a common space: for each of the experimental scan sessions, the first volume of the first run was used as a template to align the functional data from that session to the anatomical data. Co-registration was was performed using FreeSurfer's manual and automated boundary-based registration tools (Greve \& Fischl, 2009). The resulting transformation matrices were then used to transform every four-dimensional functional volume into a common space, using FSL FLIRT (Jenkinson, Bannister, Brady, \& Smith, 2002; Jenkinson \& Smith, 2001). Next, motion correction was performed using FSL MCFLIRT (Jenkinson et al., 2002), without spatial smoothing, with a final sinc interpolation stage, and 12 degrees of freedom. Finally, slow drifts in the data were removed using a high-pass filter $(1 / 40 \mathrm{~Hz}$ cutoff). No additional spatial smoothing was performed.

The above steps were performed for all functional runs, including the main working memory task (see Task: Main Working Memory), spatial working memory mapping (see Task: Spatial Working Memory Mapping), sensorimotor cortex localizer (see Task: Sensorimotor Cortex Localizer) and spatial localizer (see Task: Spatial Localizer). Following this initial pre-processing, for all run types, we normalized the time series data by z-scoring each voxel's signal across each entire scan run (this and all subsequent analyses were performed in Matlab 2018b). Deconvolution (see Analysis: Univariate) of main task data was performed on this continuous z-scored data. Next, we epoched the data based on the start time of each trial. Since trial events were jittered slightly with respect to TR onsets, we rounded trial start times to the nearest TR. This epoched data was used for time-resolved decoding analyses (Figure 2C, Figure 3B, Supplementary Figures 2 and 4). For the time-averaged analyses (Figure 2B, Figure 3A, Figure 4, Supplementary Figure 3), we obtained a single estimate of each voxel's response during each trial. For the main task, we obtained this value using an average of the timepoints from 10-16 TRs (8-12.8s) after trial onset, which falls in the delay period of the task. For the spatial working memory mapping task, we used 
an average of the timepoints from 6-12 TRs (4.8-9.6s) after trial onset, which falls in the delay period of this task. For the spatial localizer task, we averaged over the timepoints from 4-7 TRs (3.2-5.6s) after stimulus onset. For the button-pressing task (see Task: Sensorimotor Cortex Localizer) we averaged over the timepoints from 4-7 TRs (3.2-5.6s) after trial onset. The data from these latter two tasks was additionally used to identify voxels based on their spatial selectivity and action selectivity, respectively (see Identifying ROIs).

Identifying Regions of Interest (ROIs). We followed previously published retinotopic mapping protocols to define the visual areas V1, V2, V3, V3AB, hV4, IPSO, IPS1, IPS2, and IPS3 (Engel, Glover, \& Wandell, 1997; Jerde \& Curtis, 2013; Sereno et al., 1995; Swisher, Halko, Merabet, McMains, \& Somers, 2007; Wandell, Dumoulin, \& Brewer, 2007; Winawer \& Witthoft, 2015). Participants performed mapping runs in which they viewed a contrast-reversing black and white checkerboard stimulus $(4 \mathrm{~Hz})$ that was configured as either a rotating wedge (10 cycles, $36 \mathrm{~s} / \mathrm{cycle})$, an expanding ring (10 cycles, $32 \mathrm{~s} / \mathrm{cycle}$ ), or a bowtie (8 cycles, $40 \mathrm{~s} / \mathrm{cycle}$ ). To increase the quality of retinotopic data from parietal regions, participants performed a covert attention task on the rotating wedge stimulus, which required them to detect contrast dimming events that occurred occasionally (on average, 1 event every 7.5 seconds) in a row of the checkerboard (mean accuracy $=74.4 \pm 3.6 \%$ ). The maximum eccentricity of the stimulus was $9.3^{\circ}$.

After mapping the individual retinotopic ROls for each participant, we used data from our spatial localizer task (see Task: Spatial Localizer) to identify voxels within each retinotopic ROI that were selective for the region of visual space in which our spatial memory positions could appear. Data from this localizer task were analyzed using a General Linear Model (GLM) implemented in FSL's FEAT (FMRI Expert Analysis Tool, version 6.00). Brain extraction (Smith, 2002) and pre-whitening (Woolrich, Ripley, Brady, \& Smith, 2001) were performed on individual runs before analysis. Predicted BOLD responses for each of a series of checkerboard wedges were generated by convolving the stimulus sequence with a canonical gamma hemodynamic response (phase $=0 \mathrm{~s}, \mathrm{~s} . \mathrm{d} .=3 \mathrm{~s}$, lag=6s). Individual runs were combined using a standard weighted fixed effects analysis. For each of the 24 possible wedge positions, we identified voxels that were significantly more activated by that position than by all other positions $(p<0.05$, false discovery rate corrected). We then merged the sets of voxels that were identified by each of these 24 tests, and used this merged map to select voxels from each retinotopic ROI for further analysis.

In addition to mapping visual ROls, we also mapped several sensorimotor ROls. We did this by intersecting data from a simple button-press task (see Task: Sensorimotor Cortex Localizer) with anatomical definitions of motor cortex. Data from the sensorimotor localizer were analyzed using a general linear model in FSL's FEAT, as described above for the spatial localizer. Predicted BOLD responses for left- and right-handed button presses were generated by convolving the stimulus sequence with a gamma hemodynamic response function (phase $=0 \mathrm{~s}, \mathrm{~s} . \mathrm{d} .=3 \mathrm{~s}$, lag=5s). We identified voxels that showed significantly more activation for the contralateral index finger than the ipsilateral index finger $(p<0.05$, false discovery rate corrected). This procedure was done separately within each hemisphere. We then defined each sensorimotor ROI by intersecting the map of above-threshold voxels with the anatomical definitions of Brodmann's areas identified by FreeSurfer's recon-all segmentation procedure (Dale et al., 1999; Fischl et al., 2008). Specifically: the functionally-defined mask was intersected with Brodmann's area (BA) 6 was used to define premotor cortex (PMC), with BA 4 to define primary motor cortex (M1), and with BA 1,2 and 3 combined to define primary somatosensory cortex (S1) (Brodmann, 1909; Fulton, 1935; Penfield \& Boldrey, 1937). Final sizes of all visual and sensorimotor motor ROls are reported in Supplementary Table 1.

Task: Main Working Memory. For all tasks described here, stimuli were projected onto a screen $21.3 \mathrm{~cm}$ wide $\times 16 \mathrm{~cm}$ high, fixed to the inside of the scanner bore just above the participant's chest. The screen 
was viewed through a tilted mirror attached to the headcoil, from a viewing distance of $49 \mathrm{~cm}$. This resulted in a maximum vertical extent (i.e. bottom to top) of $18.5^{\circ}$, and a maximum vertical eccentricity (i.e. central fixation to top) of $9.3^{\circ}$. The background was always a mid-gray color, and the fixation point was always a black circle with radius $0.2^{\circ}$. All stimuli were generated using Ubuntu 14.04, Matlab 2017b, and the Psychophysics toolbox (Brainard, 1997; Kleiner et al., 2007).

Each trial of the main working memory task began with the fixation point turning green for $750 \mathrm{~ms}$, to alert the participant that the spatial memory target was about to appear. Next, a white target dot (radius $=0.15^{\circ}$ ) appeared for $500 \mathrm{~ms}$ at a pseudo-random position on an imaginary ring $7^{\circ}$ away from fixation (details on target positions given two paragraphs down). Participants were required to remember the precise position of this target dot. After presentation of this spatial memory target, the fixation point turned back to black for 1 second, then turned either red or blue for 2 seconds. This color cue indicated to the participant whether the current trial was an "informative" or "uninformative" trial (see next paragraph for explanation of the conditions). Next, a disk stimulus appeared for 1 second. This stimulus consisted of a circle $9.7^{\circ}$ in radius, divided into two equal halves, with each side a different shade of gray (visibly lighter and darker relative to the mean-gray background; see Figure $1 \mathrm{~A}$ ). The disk could be rotated about its center by an amount between $1^{\circ}$ and $360^{\circ}$. To avoid the disk overlapping with the fixation point, an aperture of radius $0.4^{\circ}$ was cut out of the middle of the disk, creating a donut-like shape. The inner and outer edges of the donut were smoothed with a $2 \mathrm{D}$ Gaussian kernel (size $=0.5^{\circ}$, sigma $=0.5^{\circ}$ ), but the boundary between the two halves of the disk was sharp. This "preview disk" stimulus was followed by a 12 second delay period. Following the delay period, a second disk stimulus appeared for 2 seconds, serving as the response probe (i.e. the "response disk"). Participants responded with a button press to indicate which side of the response disk the memory target had been presented on (i.e. whether the target dot fell within the light gray or dark gray side of the response disk).

For trials in the "informative" condition, the orientation of the response disk was identical to that of the preview disk. For trials in the "uninformative" condition, the orientation of the response disk was random (and unrelated to the orientation of the preview disk). Thus, for the informative condition, participants had complete knowledge of the required action as soon as the preview disk appeared, but for the uninformative condition, they had no ability to anticipate the required action. Participants were instructed not to make any physical finger movements until the response disk appeared. Responses were always made with the left or right index finger, with the mapping between shade of gray (light or dark) and finger (left or right index finger) counter-balanced across sessions within each participant. Trials of the two task conditions (i.e. informative/uninformative) were randomly interleaved within every scan run. At the start of each scan run, the participant was shown an instruction screen which reminded them of the color/condition mapping and the shade of gray/finger mapping that was in effect for that session. The mapping between color (red/blue) and condition was counter-balanced across participants, but fixed within a given participant.

Each run of the main task consisted of 20 trials, with each trial followed by an inter-trial interval jittered randomly within a range of 1-5 seconds. The total length of each run was 466 seconds. Participants performed 10 runs of this task per scan session, and completed a total of 20 runs (or 400 trials) across two separate scan sessions. All counter-balancing was performed at the session level: within each session, there were 100 trials of each condition (informative or uninformative), and on each of these 100 trials the memory target was presented at a unique polar angle position. Specifically, target positions were sampled uniformly along a $360^{\circ}$ circle, with a minimum spacing of $3.6^{\circ}$ between possible target positions. The orientation of the response disk (which determined the spatial boundary to which the memory target position was compared) also took 100 unique and uniformly spaced values along a $360^{\circ}$ circle within each condition. The possible disk orientations were shifted by $1.8^{\circ}$ relative to the possible spatial memory 
positions, so that the memory position was never exactly on the boundary of the disk. To ensure that the joint distribution of memory positions and boundary positions was close to uniform, we broke the 100 possible positions along the $360^{\circ}$ circle into 10 bins of 10 positions each. Across all 100 trials of each condition, each combination of the bin for spatial memory position and the bin for boundary orientation was used once. For the informative condition, the preview disk always took on the same orientation as the response disk. For the uninformative condition, the preview disk was assigned a random orientation, using the same 100 orientations used for the response disk but in a random order. Finally, trials for both task conditions were randomly shuffled and split evenly into 10 runs. As a result, task condition, memory target position, and response disk orientation were balanced across each session, but not balanced within individual runs.

To encourage participants to encode the spatial positions with high precision, we rewarded participants monetarily for correct performance on "hard" trials on which the spatial memory target was close to the boundary. These "hard" trials were identified as those where the spatial memory item and the boundary belonged in the same bin, according to the angular position bins described above. Participants received $\$ 1$ for correct performance on each "hard" trial, for a maximum of $\$ 40$. Across participants, the average bonus received was $\$ 32.83 \pm 2.86$.

Task: Spatial Working Memory Mapping. Participants also performed an additional working memory task while in the scanner, which served as training data for our classification analyses (see Analysis: Spatial Position Decoding). Identical to the main working memory task, each trial began with the fixation point briefly turning green $(750 \mathrm{~ms})$, followed by a spatial memory target item $(500 \mathrm{~ms})$ at a random position (at $7^{\circ}$ from fixation). The disappearance of the target was followed by a 12 second delay period, after which a white probe dot (radius $=0.15^{\circ}$ ) appeared at a random position (independent from the target position, also at $7^{\circ}$ from fixation). Participants moved this probe dot around an invisible circle to match the position at which the memory target had been presented. Participants used the four fingers of their right hand to press different buttons that corresponded to fast $\left(120^{\circ} / \mathrm{s}\right)$ or slow $\left(40^{\circ} / \mathrm{s}\right)$ movement of the probe dot in a counter-clockwise or clockwise direction. This response period lasted 3 seconds, and the final position of the probe dot was taken as the participant's response.

Each run of the spatial working memory mapping task consisted of 20 trials, with trials separated by an inter-trial interval jittered randomly within a range of 1-5 seconds. The total run length was 406 seconds. Participants performed 10 runs of this task in total, collected during a single scanning session. Across all 200 trials of the task, the spatial position of memory targets took on 200 distinct values uniformly spaced within a $360^{\circ}$ space (i.e. $1.8^{\circ}$ apart). To ensure a fairly even sampling of this space within individual runs, we binned these 200 possible positions into 20 bins of 10 positions each, and generated a sequence where each run sampled from each bin once. The random starting position of the probe dot on each trial was generated using an identical procedure, but independently of the memory targets, so that there was no association between the position of the spatial memory target and the probe start position. The absolute average angular error across 6 participants on this task was $7.0^{\circ} \pm 0.9^{\circ}$.

Task: Spatial Localizer. We ran a spatial localizer task for two purposes, namely, to identify voxels having spatial selectivity within the region of space spanned by the memory positions (see Identifying ROIs), and to serve as training data for our classification analyses (see Analysis: Spatial Position Decoding). In this task, participants viewed black and white checkerboard wedges flickering at a rate of $4 \mathrm{~Hz}$. Wedges had a width of $15^{\circ}$ (polar angle), spanned an eccentricity range of $4.4^{\circ}-9.3^{\circ}$ (visual angle), and were positioned at 24 different positions around an imaginary circle. Possible wedge center positions were offset from the cardinal axes by $7.5^{\circ}$ (i.e., a wedge was never centered on the horizontal or vertical meridian). Each run included 4 wedge presentations at each position, totaling 96 trials. The sequence of positions was random 
with the constraint that consecutively presented wedges never appeared in the same quadrant. Trials were 3 seconds each, and were not separated by an inter-trial interval. The total run length was 313 seconds. During each run, participants performed a change-detection task at fixation, where they responded with a button press any time the fixation point increased or decreased in brightness. A total of 20 brightness changes occurred in each run, at times that were random with respect to trial onsets. The magnitude of brightness changes was adjusted manually at the start of each run to control the difficulty. Average detection performance (hit rate) was $76.7 \pm 4.2 \%$. participants performed between 8 and 16 total runs of this task. For some participants, some of these runs were collected as part of a separate experiment.

Task: Sensorimotor Cortex Localizer. Participants also performed a sensorimotor cortex localizer task in the scanner. Analogous to our use of the spatial localizer task, this data served a dual purpose: it was used to identify ROIs in motor and somatosensory cortex that were selective for contralateral index finger button presses (see Identifying ROIs), and as a training set for one of our classification analyses (see Analysis: Action Decoding). Participants attended a black fixation point $\left(0.2^{\circ}\right)$, and responded to brief $(1000 \mathrm{~ms})$ color changes of the fixation point by pressing a button with their left or right index finger. The fixation dot changed to either magenta or cyan to indicate which finger should be used, and each color change was separated by an inter-trial interval randomly jittered in the range of 2-6 seconds. Each run was 319 seconds long, and included 60 total trials (i.e. 60 button presses), with 30 trials for each finger randomly interleaved. The color/finger mapping was switched on alternating runs. Participants were instructed to respond as quickly as possible to each color change. Average performance on this task was $92.8 \pm 3.0 \%$ correct, and average behavioral response time was $530 \pm 23 \mathrm{~ms}$. Each participant performed 6 runs of this task.

Analysis: Univariate. In order to estimate a hemodynamic response function (HRF) for each voxel during each condition (Figure 1D, Supplementary Figure 1) we used linear deconvolution. We constructed a finite impulse response model (Dale, 1999) that included a series of regressors for trials in each task condition: one regressor marking the onset of a spatial memory target item, followed by a series of temporally shifted versions of that regressor (to model the BOLD response at each subsequent time point in the trial). The model also included a constant regressor for each of the 20 total runs. The data used as input to this GLM was z-scored on a voxel-by-voxel basis within runs (see Pre-Processing of FMRI Data). Estimated HRFs for the two conditions were averaged across all voxels within each ROI. To evaluate whether the mean BOLD signal in each ROI differed significantly between conditions, we used a permutation test. First, for each ROI and timepoint, we computed a Wilcoxon signed rank statistic comparing the activation values for each participant from condition 1 to the activation values for each participant from condition 2 . Then, we performed 1000 iterations of shuffling the condition labels within each participant (swapping the condition labels for each participant with $50 \%$ probability). We then computed a signed rank statistic from the shuffled values on each iteration. Finally, we computed a two-tailed p-value for each ROI and timepoint by computing the number of iterations on which the shuffled signed rank statistic was $>=$ the real statistic, and the number of iterations on which the shuffled statistic was $<=$ the real statistic, and taking the smaller of these two values. We obtained the final $p$-value by dividing this value by the number of iterations and multiplying by 2 .

Analysis: Spatial Position Decoding. We used linear classification to measure representations of remembered spatial position information in each visual and sensorimotor ROI during the main working memory task. Since we were interested in assessing the coding format of memory representations, we separately performed decoding using three different approaches. In the first approach (Figure 2, Supplementary Figure 2), we trained the decoder on independent data measured while participants were remembering a given spatial position (see Task: Spatial Working Memory Mapping). In the second 
approach (Figure 4A), we trained the decoder on independent data collected when participants were perceiving a physical stimulus at a given spatial position (see Task: Spatial Localizer). In the final method (Supplementary Figure 3), we used cross-validation to train and test our decoder using data from within each condition of the main working memory task.

Before performing each of these classification methods, we first mean-centered the voxel activation pattern from each trial in each task by subtracting the mean across all voxels from each trial. Next, we binned all trials of the main working memory task (see Task: Main Working Memory) and the spatial working memory mapping task (see Task: Spatial Working Memory Mapping) into 8 angular position bins that each spanned $45^{\circ}$, with the first bin centered at $0^{\circ}$. Trials from the spatial localizer task (see Task: Spatial Localizer) were binned into 8 angular position bins that each spanned $60^{\circ}$ (i.e. the bins were slightly overlapping and some wedge positions contributed to multiple bins; similar results were obtained using bins that were entirely non-overlapping). We then performed binary classification between pairs of bins that were $180^{\circ}$ apart (see Figure $2 \mathrm{~A}$ ), using a linear classifier based on the normalized Euclidean distance (for more details see Henderson \& Serences, 2019). This meant that we constructed four separate binary classifiers, each operating on approximately $1 / 4$ of the data, with a chance decoding value of $50 \%$. We then averaged across the four binary classifiers to get a single index of classification accuracy for each ROI and task condition.

For the results shown in Figure 2 and Supplementary Figure 2, the training set for these classifiers consisted of data from the spatial working memory mapping task (averaged within a fixed time window during the delay period 4.8-9.6 s after trial onset), and the test set consisted of data from the main working memory task (either averaged over a window of 8-12.8 seconds after trial onset, or at each individual TR following trial onset). For the results based on sensory-driven responses (Figure 4A), the training set consisted of data from the spatial localizer task (averaged over a window 3.2-5.6 s after stimulus onset), and the test set consisted of data from the main working memory task (same time window defined above). For the within-condition analyses (Supplementary Figure 3), the training and testing sets both consisted of data from a single condition in the main working memory task (either informative or uninformative; same time window defined above). Each binary classifier was cross-validated by leaving out two trials at a time for testing (leaving out one trial per class ensures the training set was always balanced), and looping over cross-validation folds so that every trial served as a test trial once.

To test whether decoding performance was significantly above chance in each ROI and condition of the main working memory task, we used a permutation test. On each of 1000 iterations, we shuffled the binary labels for the training set, trained a classifier on this shuffled data, and then computed how well this decoder predicted the binary labels in the test set. For each iteration, we then computed a Wilcoxon signed rank statistic comparing the $\mathrm{N}$ participants' real decoding values to the $\mathrm{N}$ participants' shuffled decoding values. A signed rank statistic greater than 0 indicated the median of the real decoding values was greater than the median of the shuffled decoding values, and a statistic less than zero indicated the median of the null decoding values was greater than the median of the real values. We obtained a onetailed $p$-value for each ROI and task condition across all participants by counting the number of iterations on which the signed rank statistic was less than or equal to zero, and dividing by the total number of iterations.

To test whether decoding performance differed significantly between the two task conditions within each ROI, we used a permutation test. First, for each ROI, we computed a Wilcoxon signed rank statistic comparing the $\mathrm{N}$ participants' decoding values from condition 1 to the $\mathrm{N}$ participants' decoding values from condition 2. Then, we performed 1000 iterations of shuffling the condition labels within each participant (swapping the condition labels for each participant with $50 \%$ probability). We then computed 
a signed rank statistic from the shuffled values. Finally, we computed a two-tailed $p$ value for each ROI by computing the number of iterations on which the shuffled signed rank statistic was $>=$ the real statistic, and the number of iterations on which the shuffled statistic was $<=$ the real statistic, and taking the smaller of these two values. We obtained the final $p$-value by dividing this value by the total number of iterations and multiplying by 2 .

The above procedures were used for all time-averaged and time-resolved decoding analyses (i.e. for time-resolved analyses we repeated the same statistical procedures at each timepoint separately). For the time-averaged decoding accuracies, we also performed a two-way repeated measures ANOVA with factors of ROI, condition, and a ROI x condition interaction (implemented using ranova.m). We performed a permutation test where we shuffled the decoding scores within each participant 1000 times, and computed an F-statistic for each effect on the shuffled data. Across all permutations, we obtained a nulldistribution of F-values for effects of ROI, condition, and the ROI x condition interaction. P-values for each effect were based on the number of times the shuffled F-statistic for that effect was greater than or equal to the real F-statistic, divided by the total number of iterations (similar to method used in Rademaker et al., 2019). F-statistics reported in the text reflect the F-statistic obtained using the real (unshuffled) data.

Analysis: Action Decoding. We performed linear classification (as above) to measure the representation of information related to left or right index finger button presses in each ROI. To assess the coding format of action representations, we separately performed decoding using two different approaches. In the first approach, we used data from the main working memory task to train and test the classifier (Figure 3, Supplementary Figure 4). Here, action classification was always done using data from one task condition at a time (i.e. informative or uninformative trials). The decoder was always trained on data from one session and tested on the other session. Because the mapping of disk side color (light or dark gray) to finger was always switched between the two sessions, this ensured that the information detected by the classifier was not related to the luminance of the half of the disk corresponding to the response finger. In the second approach, we trained the classifier using data from a separate task during which participants were physically pressing a button (see Task: Sensorimotor Cortex Localizer), and we tested using data from the main working memory task (Figure 4B). Irrespective of the training-testing approach used, classification was based on trials labeled according to the finger (left or right index) that corresponded to the correct response. This means that all trials were included (also those where the incorrect button, or no button, was pressed), which ensures the training set for the classifier was balanced. Note that qualitatively similar results were obtained when using correct trials only, or when using the participant's actual response as the label for the decoder.

The above procedure was used for both time-averaged (Figure $3 \mathrm{~A}$ and $4 \mathrm{~B}$ ) and time-resolved action decoding (Figure 3B, Supplementary Figure 4). For time-averaged decoding, single trial responses were obtained by averaging over specified time windows after trial onset (8-12.8s for the main working memory task; 3.2-5.6s for the sensorimotor cortex localizer). For time-resolved decoding on the main working memory task data, the training and testing set each consisted of data from the TR of interest (but from different sessions, as described above). All statistical tests on the results of action decoding were performed in an identical manner to the statistics on the results of spatial decoding (see Analysis: Spatial Position Decoding). 


\section{Supplementary Figures}
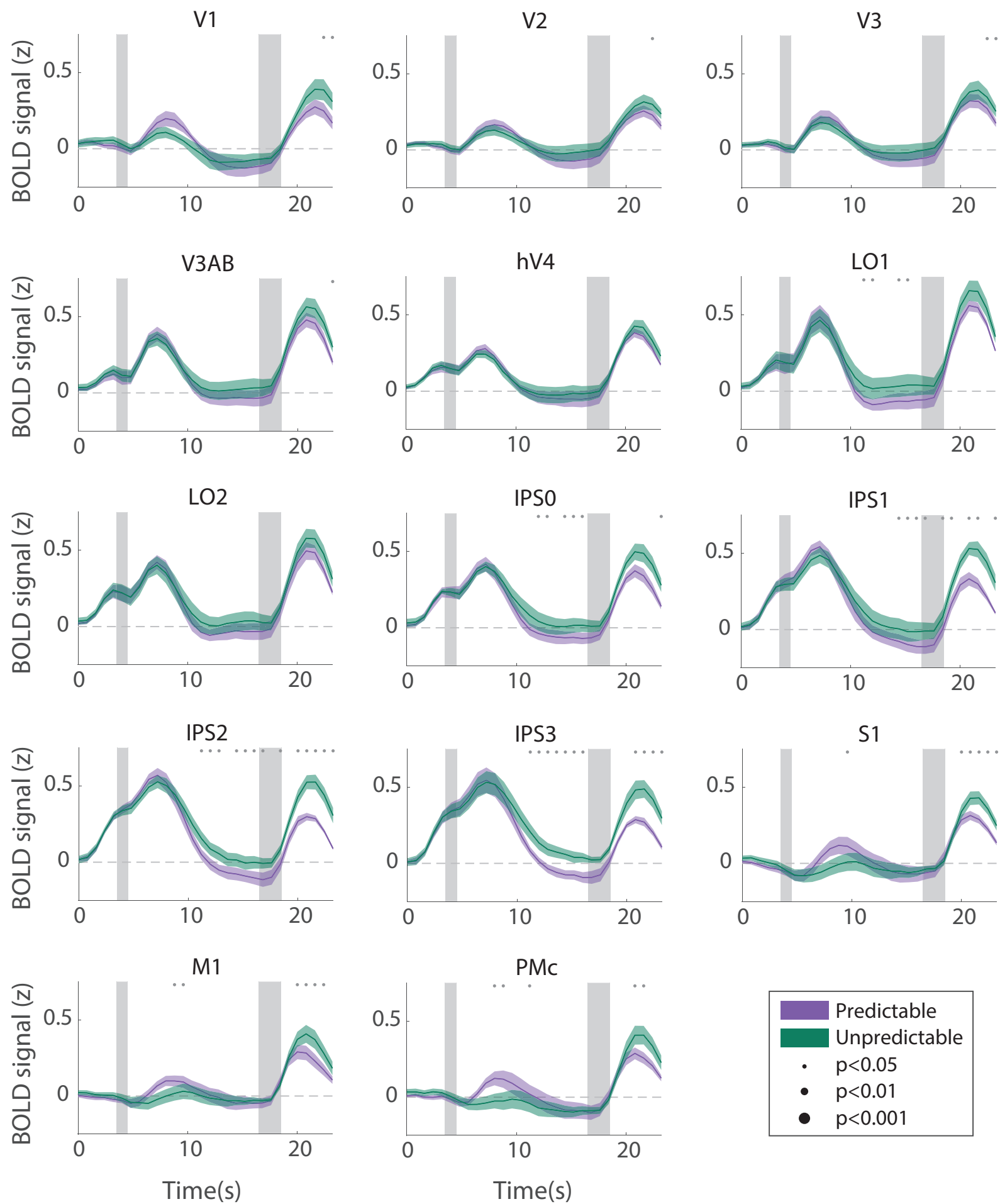

Supplementary Figure 1. Hemodynamic response function in each ROI during the informative (purple) and uninformative (green) conditions, full set of ROls. Timepoint zero indicates the time of target onset; shaded gray rectangles indicate the time periods when the "preview" disk was onscreen (3.5-4.5 sec) and when the response disk was onscreen (16.5-18.5 sec). Shaded error bars represent \pm 1 SEM across participants. Gray dots indicate timepoints showing a significant condition difference, evaluated using a Wilcoxon signed-rank test with permutation, all p-values <0.05; see Methods, Analysis: Univariate for details). 

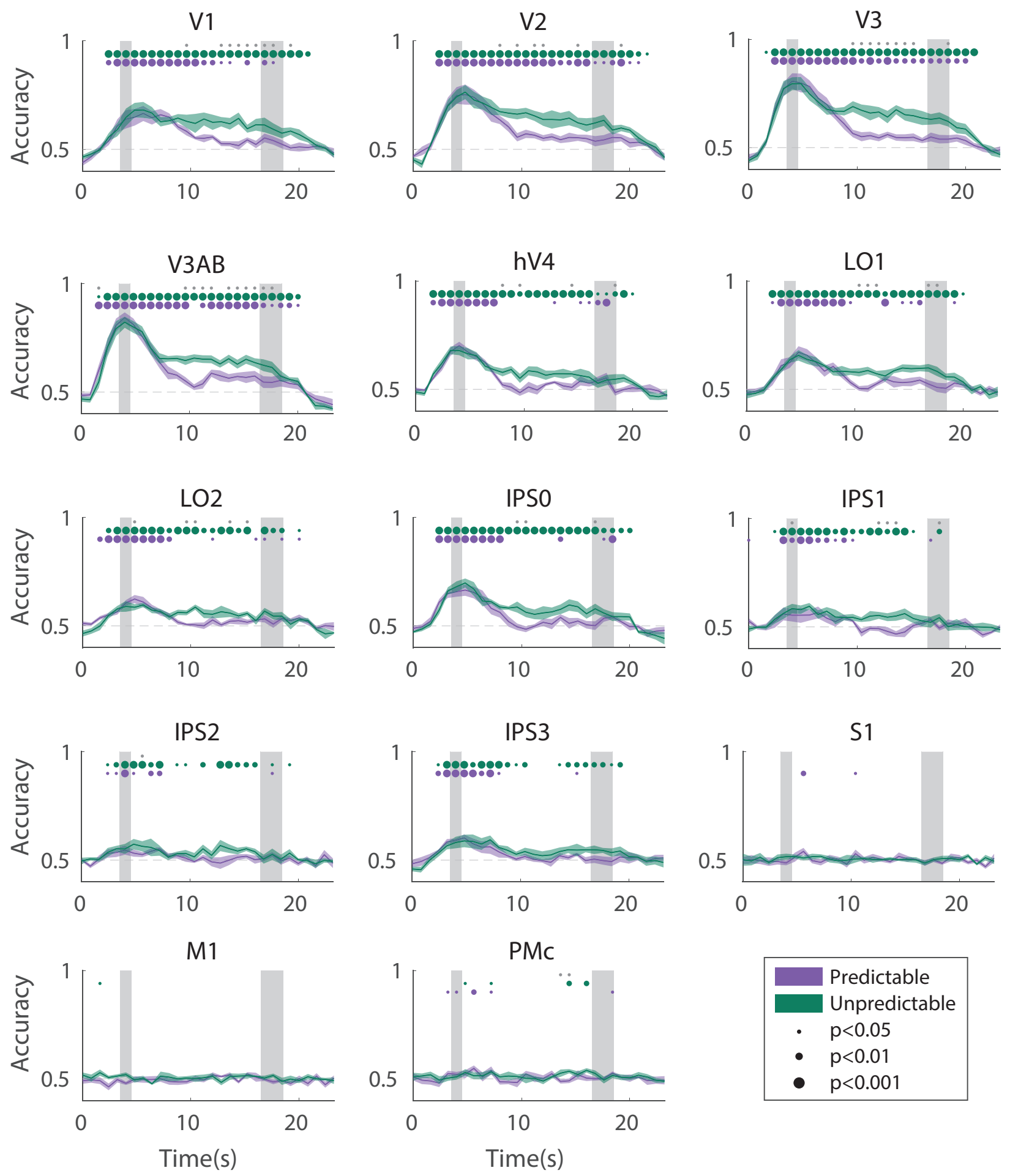

Supplementary Figure 2. Time-resolved spatial decoding accuracy in every ROI. All decoding was done using the spatial working memory mapping task as a training set (see Methods, Analysis: Spatial Position Decoding for details). Timepoint zero indicates the time of target onset; shaded gray rectangles indicate the periods of time when the "preview" disk was onscreen (3.5-4.5 sec) and when the response disk was onscreen $(16.5-18.5 \mathrm{sec})$. Shaded error bars represent $\pm 1 \mathrm{SEM}$ across participants, colored dots indicate significance of decoding within each condition, and gray dots indicate significant condition differences, with dot sizes reflecting significance levels. 


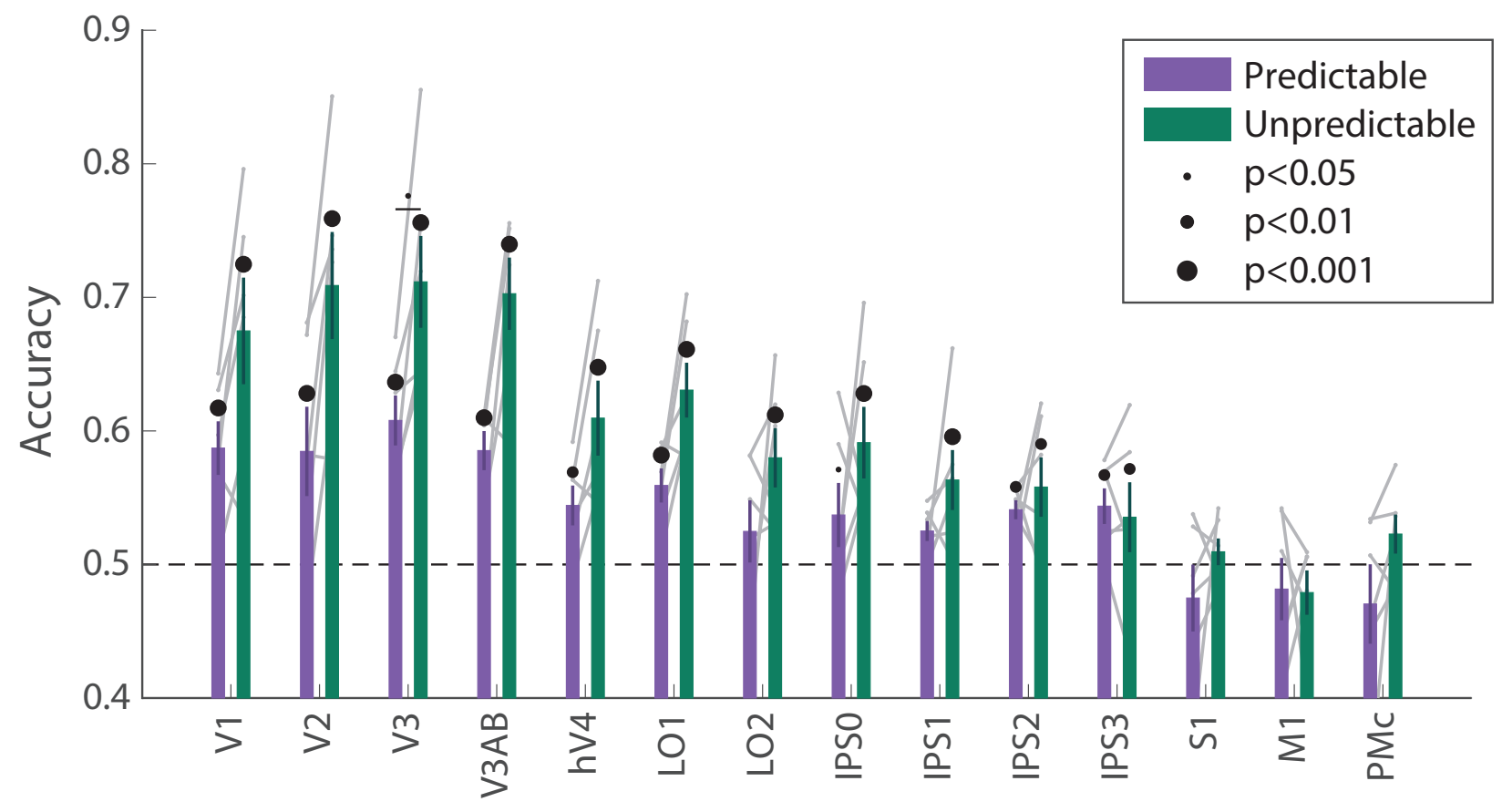

Supplementary Figure 3. Spatial decoding performance differs across conditions, even when training and testing a decoder within each task condition separately. See Methods, Analysis: Spatial Position Decoding for details on classification procedure. Notably, within-condition spatial decoding showed a highly similar pattern of results to the analysis using the independent training set (Figure $2 B$ ), though the condition differences were slightly smaller (main effect of ROI: $F_{(13,65)}=12.873, p<0.001$; main effect of condition: $F_{(1,5)}=11.461, p=0.017 ; R O I \times$ Condition interaction: $F_{(13,65)}=2.581, p=0.011 ; p$-values obtained using permutation test). Error bars reflect \pm 1 SEM across participants, and light gray lines indicate individual participants. Dots above bars and pairs of bars indicate the statistical significance of decoding within each condition, and of condition differences, respectively, both evaluated using non-parametric statistics. Dot sizes reflect significance level. 

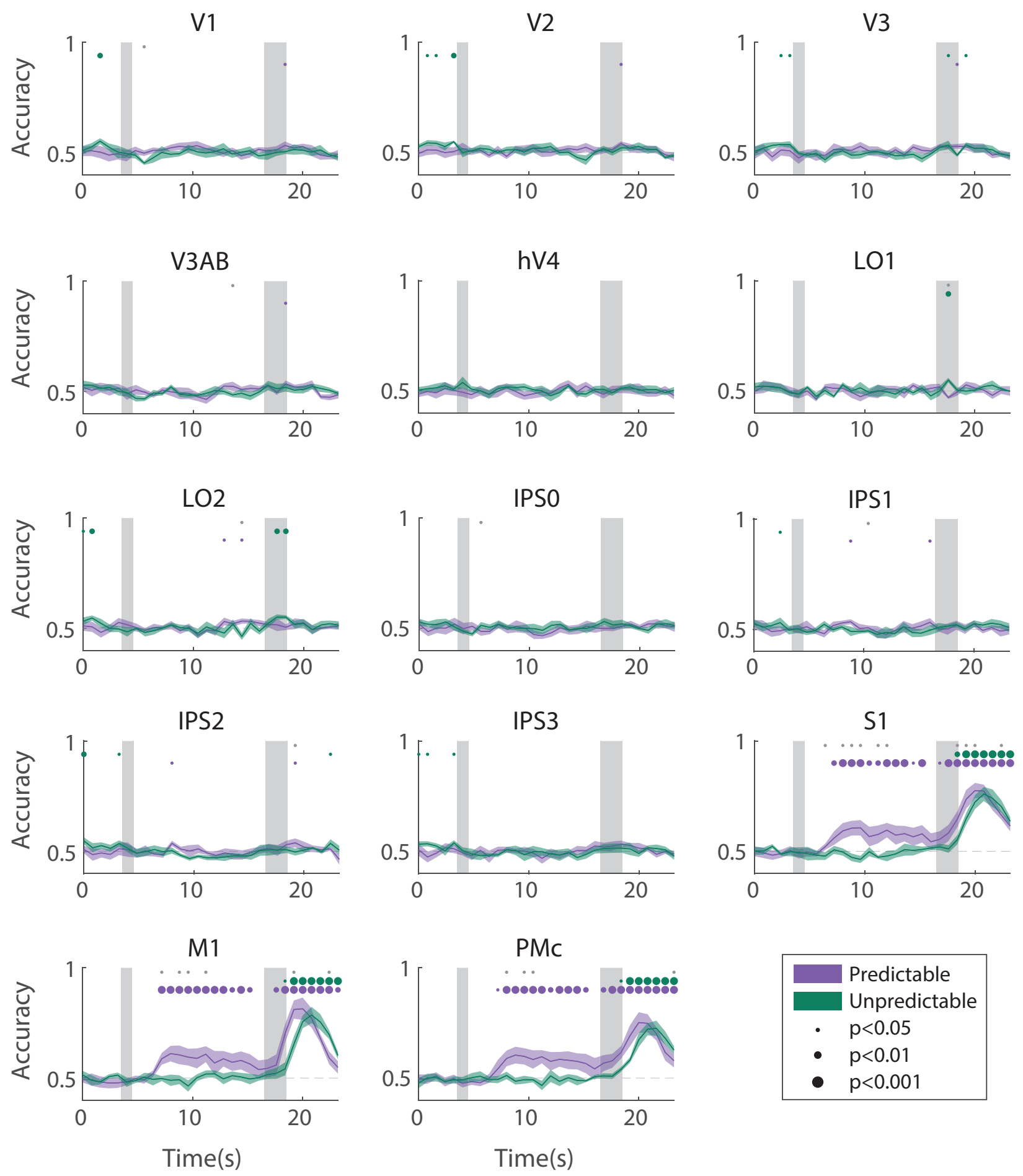

Supplementary Figure 4. Time-resolved action decoding accuracy in every ROI. All decoding was done using data from the same task condition for training and testing (see Methods, Analysis: Action Decoding for details). Timepoint zero indicates the time of target onset; shaded gray rectangles indicate the periods of time when the "preview" disk was onscreen (3.5-4.5 sec) and when the response disk was onscreen $(16.5-18.5 \mathrm{sec})$. Shaded error bars represent \pm 1 SEM across participants, colored dots indicate significance of decoding within each condition, and gray dots indicate significant condition differences, with dot sizes reflecting significance levels. 


\section{Supplementary Tables}

\begin{tabular}{|c|c|c|c|c|c|c|c|c|c|c|c|c|}
\hline \multirow[b]{3}{*}{ V1 } & \multicolumn{2}{|c|}{ S01 } & \multicolumn{2}{|c|}{ S02 } & \multicolumn{2}{|c|}{ S03 } & \multicolumn{2}{|c|}{ S04 } & \multicolumn{2}{|c|}{ S05 } & \multicolumn{2}{|c|}{ S06 } \\
\hline & Ih & rh & Ih & rh & Ih & rh & Ih & rh & Ih & rh & Ih & rh \\
\hline & 627 & 673 & 514 & 429 & 646 & 552 & 564 & 644 & 370 & 393 & 626 & 800 \\
\hline V2 & 682 & 689 & 409 & 430 & 398 & 542 & 580 & 623 & 362 & 425 & 527 & 529 \\
\hline V3 & 503 & 511 & 475 & 486 & 517 & 595 & 588 & 480 & 329 & 409 & 614 & 402 \\
\hline V3AB & 311 & 370 & 465 & 459 & 350 & 317 & 287 & 301 & 281 & 292 & 448 & 491 \\
\hline hV4 & 198 & 169 & 324 & 347 & 294 & 358 & 252 & 185 & 330 & 220 & 105 & 114 \\
\hline L01 & 162 & 200 & 104 & 108 & 132 & 107 & 315 & 177 & 133 & 101 & 249 & 64 \\
\hline LO2 & 282 & 257 & 107 & 50 & 77 & 88 & 136 & 167 & 115 & 72 & 79 & 103 \\
\hline IPSO & 117 & 250 & 263 & 260 & 209 & 206 & 183 & 114 & 97 & 143 & 357 & 249 \\
\hline IPS1 & 99 & 172 & 233 & 132 & 123 & 116 & 91 & 154 & 40 & 36 & 158 & 178 \\
\hline IPS2 & 89 & 205 & 295 & 178 & 63 & 97 & 144 & 145 & 36 & 3 & 192 & 191 \\
\hline IPS3 & 188 & 183 & 227 & 265 & 174 & 109 & 144 & 143 & 49 & 94 & 219 & 152 \\
\hline S1 & 537 & 672 & 194 & 358 & 476 & 868 & 182 & 1350 & 183 & 401 & 824 & 495 \\
\hline M1 & 154 & 215 & 97 & 164 & 103 & 234 & 143 & 410 & 66 & 63 & 272 & 122 \\
\hline PMC & 111 & 228 & 45 & 136 & 84 & 448 & 115 & 547 & 16 & 10 & 302 & 133 \\
\hline
\end{tabular}

Supplementary Table 1. Number of voxels in each ROI for each participant and hemisphere. Sizes of retinotopic visual ROIs (V1IPS3) are after thresholding with a spatial localizer (see Methods, Task: Spatial Localizer). S1, M1, and PMC were defined using a button-pressing task (see Methods, Task: Sensorimotor Cortex Localizer). All analyses in this paper were done using bilateral ROIs (i.e. concatenating the left and right hemispheres of each ROI). 


\section{$\underline{\text { References }}$}

Albers, A. M., Kok, P., Toni, I., Dijkerman, H. C., \& De Lange, F. P. (2013). Shared representations for working memory and mental imagery in early visual cortex. Current Biology, 23(15), 1427-1431. https://doi.org/10.1016/j.cub.2013.05.065

Andersson, J. L. R., Skare, S., \& Ashburner, J. (2003). How to correct susceptibility distortions in spinecho echo-planar images: Application to diffusion tensor imaging. Neurolmage, 20(2), 870-888. https://doi.org/10.1016/S1053-8119(03)00336-7

Ariani, G., Pruszynski, J. A., \& Diedrichsen, J. (2020). Motor planning brings human primary somatosensory cortex into movement-specific preparatory states. BioRxiv, 2020.12.17.423254. https://doi.org/10.1101/2020.12.17.423254

Awh, E., \& Jonides, J. (2001). Overlapping mechanisms of attention and spatial working memory. Trends in Cognitive Sciences, 5(3), 119-126. Retrieved from http://www.ncbi.nlm.nih.gov/pubmed/11239812

Baddeley, A. D., \& Hitch, G. (1974). Working memory. Psychology of Learning and Motivation - Advances in Research and Theory, 8(C), 47-89. https://doi.org/10.1016/S0079-7421(08)60452-1

Barbosa, J., Soldevilla, D. L., \& Compte, A. (2021). Unattended short-term memories are maintained in active neural representations. PsyArXiv. https://doi.org/10.31234/OSF.IO/QV6FU

Bettencourt, K. C., \& Xu, Y. (2015). Decoding the content of visual short-term memory under distraction in occipital and parietal areas. Nature Neuroscience, 19(1), 150-157. https://doi.org/10.1038/nn.4174

Boettcher, S. E. P., Gresch, D., Nobre, A. C., \& Van Ede, F. (2021). Output planning at the input stage in visual working memory. Science Advances, 7(13). https://doi.org/10.1126/sciadv.abe8212

Boynton, G. M. (2011). Spikes, BOLD, attention, and awareness: A comparison of electrophysiological and fMRI signals in V1. Journal of Vision, 11(5), 12-12. https://doi.org/10.1167/11.5.12

Brainard, D. H. (1997). The Psychophysics Toolbox. Spatial Vision, 10(4), 433-436. Retrieved from http://www.ncbi.nlm.nih.gov/pubmed/9176952

Brodmann, K. (1909). Physiology of the cortex as an organ. In Brodmann's Localisation in the Cerebral Cortex (pp. 239-262). https://doi.org/10.1007/0-387-26919-3_10

Calderon, C. B., Van Opstal, F., Peigneux, P., Verguts, T., \& Gevers, W. (2018). Task-Relevant Information Modulates Primary Motor Cortex Activity Before Movement Onset. Frontiers in Human Neuroscience, 12, 93. https://doi.org/10.3389/fnhum.2018.00093

Christophel, T. B., Hebart, M. N., \& Haynes, J.-D. (2012). Decoding the contents of visual short-term memory from human visual and parietal cortex. The Journal of Neuroscience : The Official Journal of the Society for Neuroscience, 32(38), 12983-12989. https://doi.org/10.1523/JNEUROSCI.018412.2012

Cisek, P., \& Kalaska, J. F. (2005). Neural correlates of reaching decisions in dorsal premotor cortex: Specification of multiple direction choices and final selection of action. Neuron, 45(5), 801-814. https://doi.org/10.1016/j.neuron.2005.01.027

Cisek, P., \& Kalaska, J. F. (2010). Neural mechanisms for interacting with a world full of action choices. 
Annual Review of Neuroscience, Vol. 33, pp. 269-298.

https://doi.org/10.1146/annurev.neuro.051508.135409

Curtis, C. E., \& D'Esposito, M. (2003). Persistent activity in the prefrontal cortex during working memory. Trends in Cognitive Sciences, 7(9), 415-423. https://doi.org/10.1016/S1364-6613(03)00197-9

Curtis, C. E., Rao, V. Y., \& D’Esposito, M. (2004). Maintenance of Spatial and Motor Codes during Oculomotor Delayed Response Tasks. Journal of Neuroscience, 24(16), 3944-3952. https://doi.org/10.1523/JNEUROSCI.5640-03.2004

D'Esposito, M. (2007). From cognitive to neural models of working memory. Philosophical Transactions of the Royal Society B: Biological Sciences, 362(1481), 761-772. https://doi.org/10.1098/rstb.2007.2086

Dale, A. M. (1999). Optimal experimental design for event-related fMRI. Human Brain Mapping, 8(2-3), 109-114. https://doi.org/10.1002/(SICI)1097-0193(1999)8:2/33.0.CO;2-W

Dale, A. M., \& Buckner, R. L. (1997). Selective averaging of rapidly presented individual trials using fMRI. Retrieved May 26, 2021, from Human Brain Mapping website: https://onlinelibrary.wiley.com/doi/10.1002/(SICI)1097-0193(1997)5:5\%3C329::AIDHBM1\%3E3.0.CO;2-5

Dale, A. M., Fischl, B., \& Sereno, M. I. (1999). Cortical surface-based analysis: I. Segmentation and surface reconstruction. Neurolmage, 9(2), 179-194. https://doi.org/10.1006/nimg.1998.0395

Donner, T. H., Siegel, M., Fries, P., \& Engel, A. K. (2009). Buildup of Choice-Predictive Activity in Human Motor Cortex during Perceptual Decision Making. Current Biology, 19(18), 1581-1585. https://doi.org/10.1016/j.cub.2009.07.066

Emrich, S. M., Riggall, A. C., La Rocque, J. J., \& Postle, B. R. (2013). Distributed patterns of activity in sensory cortex reflect the precision of multiple items maintained in visual short-term memory. Journal of Neuroscience, 33(15), 6516-6523. https://doi.org/10.1523/JNEUROSCI.5732-12.2013

Engel, S., Glover, G. H., \& Wandell, B. A. (1997). Retinotopic organization in human visual cortex and the spatial precision of functional MRI. Cerebral Cortex, 7(2), 181-192. https://doi.org/10.1093/cercor/7.2.181

Ester, E. F., Rademaker, R. L., \& Sprague, T. C. (2016). How do visual and parietal cortex contribute to visual short-term memory? ENeuro, 3(2), 150-157. https://doi.org/10.1523/ENEURO.0041-16.2016

Ester, E. F., Serences, J. T., \& Awh, E. (2009). Spatially global representations in human primary visual cortex during working memory maintenance. Journal of Neuroscience, 29(48), 15258-15265. https://doi.org/10.1523/JNEUROSCl.4388-09.2009

Ester, E. F., Sprague, T. C., \& Serences, J. T. (2015). Parietal and Frontal Cortex Encode Stimulus-Specific Mnemonic Representations during Visual Working Memory. Neuron, 87(4), 893-905. https://doi.org/10.1016/j.neuron.2015.07.013

Fischl, B., Rajendran, N., Busa, E., Augustinack, J., Hinds, O., Yeo, B. T. T., ... Zilles, K. (2008). Cortical Folding Patterns and Predicting Cytoarchitecture. Cerebral Cortex August, 18, 1973-1980. https://doi.org/10.1093/cercor/bhm225

Fulton, J. F. (1935). A note on the definition of the "motor" and "premotor" areas. Brain, 58(2), 311-316. 
Funahashi, S., Bruce, C. J., \& Goldman-Rakic, P. S. (1989). Mnemonic coding of visual space in the monkey's dorsolateral prefrontal cortex. Journal of Neurophysiology, 61(2), 331-349. https://doi.org/10.1016/j.neuron.2012.12.039

Funahashi, S., Chafee, M. V., \& Goldman-Rakic, P. S. (1993). Prefrontal neuronal activity in rhesus monkeys performing a delayed anti-saccade task. Nature, 365(6448), 753-756. https://doi.org/10.1038/365753a0

Fuster, J. M., \& Alexander, G. E. (1971). Neuron activity related to short-term memory. Science (New York, N.Y.), 173(3997), 652-654. https://doi.org/10.1126/SCIENCE.173.3997.652

Gazzaley, A., \& Nobre, A. C. (2012, February 1). Top-down modulation: Bridging selective attention and working memory. Trends in Cognitive Sciences, Vol. 16, pp. 129-135. https://doi.org/10.1016/j.tics.2011.11.014

Goense, J. B. M., \& Logothetis, N. K. (2008). Neurophysiology of the BOLD fMRI Signal in Awake Monkeys. Current Biology, 18(9), 631-640. https://doi.org/10.1016/j.cub.2008.03.054

Goldman-Rakic, P. . S. (1995). Cellular basis of working memory. Neuron, 14(3), 477-485. https://doi.org/10.1016/0896-6273(95)90304-6

Greve, D. N., \& Fischl, B. (2009). Accurate and robust brain image alignment using boundary-based registration. Neurolmage, 48(1), 63-72. https://doi.org/10.1016/j.neuroimage.2009.06.060

Harrison, S. A., \& Tong, F. (2009). Decoding reveals the contents of visual working memory in early visual areas. Nature, 458(7238), 632-635. https://doi.org/10.1038/nature07832

Henderson, M., \& Serences, J. T. (2019). Human frontoparietal cortex represents behaviorally relevant target status based on abstract object features. Journal of Neurophysiology, 121(4). https://doi.org/10.1152/jn.00015.2019

lamshchinina, P., Christophel, T. B., Gayet, S., \& Rademaker, R. L. (n.d.). Essential considerations for exploring visual working memory storage in the human brain. Visual Cognition.

lamshchinina, P., Christophel, T. B., Gayet, S., \& Rademaker, R. L. (2021). Essential considerations for exploring visual working memory storage in the human brain. Visual Cognition, 1-12. https://doi.org/10.1080/13506285.2021.1915902

Jenkinson, M., Bannister, P., Brady, M., \& Smith, S. (2002). Improved optimization for the robust and accurate linear registration and motion correction of brain images. Neurolmage, 17(2), 825-841. https://doi.org/10.1016/S1053-8119(02)91132-8

Jenkinson, M., Beckmann, C. F., Behrens, T. E. J., Woolrich, M. W., \& Smith, S. M. (2012). Fsl. Neurolmage, 62(2), 782-790. https://doi.org/10.1016/j.neuroimage.2011.09.015

Jenkinson, M., \& Smith, S. (2001). A global optimisation method for robust affine registration of brain images. Medical Image Analysis, 5(2), 143-156. https://doi.org/10.1016/S1361-8415(01)00036-6

Jerde, T. A., \& Curtis, C. E. (2013). Maps of space in human frontoparietal cortex. Journal of Physiology Paris, 107(6), 510-516. https://doi.org/10.1016/j.jphysparis.2013.04.002

Klein-Flügge, M. C., \& Bestmann, S. (2012). Time-dependent changes in human corticospinal excitability reveal value-based competition for action during decision processing. Journal of Neuroscience, 32(24), 8373-8382. https://doi.org/10.1523/JNEUROSCI.0270-12.2012 
Kleiner, M., Brainard, D. H., Pelli, D. G., Broussard, C., Wolf, T., \& Niehorster, D. (2007). What's new in Psychtoolbox-3? A free cross-platform toolkit for psychophysiscs with Matlab and GNU/Octave. In Cognitive and Computational Psychophysics (Vol. 36). https://doi.org/10.1068/v070821

Leavitt, M. L., Mendoza-Halliday, D., \& Martinez-Trujillo, J. C. (2017). Sustained Activity Encoding Working Memories: Not Fully Distributed. Trends in Neurosciences, 40(6), 328-346. https://doi.org/10.1016/j.tins.2017.04.004

Lee, S. H., Kravitz, D. J., \& Baker, C. I. (2013). Goal-dependent dissociation of visual and prefrontal cortices during working memory. Nature Neuroscience, 16(8), 997-999. https://doi.org/10.1038/nn.3452

Lewis-Peacock, J. A., Drysdale, A. T., Oberauer, K., \& Postle, B. R. (2012). Neural evidence for a distinction between short-term memory and the focus of attention. Journal of Cognitive Neuroscience, 24(1), 61-79. https://doi.org/10.1162/jocn_a_00140

Logothetis, N. K., Pauls, J., Augath, M., Trinath, T., \& Oeltermann, A. (2001). Neurophysiological investigation of the basis of the fMRI signal. Nature, 412(6843), 150-157. https://doi.org/10.1038/35084005

Logothetis, N. K., \& Wandell, B. A. (2004). INTERPRETING THE BOLD SIGNAL. Annu. Rev. Physiol, 66, 735769. https://doi.org/10.1146/annurev.physiol.66.082602.092845

Lorenc, E. S., Sreenivasan, K. K., Nee, D. E., Vandenbroucke, A. R. E., \& D’Esposito, M. (2018). Flexible coding of visual working memory representations during distraction. Journal of Neuroscience, 38(23), 5267-5276. https://doi.org/10.1523/JNEUROSCI.3061-17.2018

Lorenc, E. S., Vandenbroucke, A. R. E., Nee, D. E., de Lange, F. P., \& D’Esposito, M. (2020). Dissociable neural mechanisms underlie currently-relevant, future-relevant, and discarded working memory representations. Scientific Reports, 10(1). https://doi.org/10.1038/s41598-020-67634-x

Mendoza-Halliday, D., Torres, S., \& Martinez-Trujillo, J. C. (2014). Sharp emergence of feature-selective sustained activity along the dorsal visual pathway. Nature Neuroscience, 17(9), 1255-1262. https://doi.org/10.1038/nn.3785

Miller, E., Erickson, C. a, \& Desimone, R. (1996). Neural mechanisms of visual working memory in prefrontal cortex of the macaque. Journal of Neuroscience, 16(16), 5154-5167. https://doi.org/10.1.1.41.2959

Myers, N. E., Stokes, M. G., \& Nobre, A. C. (2017, June 1). Prioritizing Information during Working Memory: Beyond Sustained Internal Attention. Trends in Cognitive Sciences, Vol. 21, pp. 449-461. https://doi.org/10.1016/j.tics.2017.03.010

Nobre, A. C., \& Stokes, M. G. (2019, October 9). Premembering Experience: A Hierarchy of Time-Scales for Proactive Attention. Neuron, Vol. 104, pp. 132-146.

https://doi.org/10.1016/j.neuron.2019.08.030

Offen, S., Schluppeck, D., \& Heeger, D. J. (2009). The role of early visual cortex in visual short-term memory and visual attention. Vision Research, 49(10), 1352-1362.

https://doi.org/10.1016/j.visres.2007.12.022

Panichello, M. F., DePasquale, B., Pillow, J. W., \& Buschman, T. J. (2019). Error-correcting dynamics in visual working memory. Nature Communications, 10(1), 1-11. https://doi.org/10.1038/s41467- 


\section{9-11298-3}

Pasternak, T., \& Greenlee, M. W. (2005). Working memory in primate sensory systems. Nature Reviews Neuroscience, 6(2), 97-107. https://doi.org/10.1038/nrn1603

Penfield, W., \& Boldrey, E. (1937). Somatic motor and sensory representation in the cerebral cortex of man as studied by electrical stimulation. In Brain (Vol. 60). https://doi.org/10.1093/brain/60.4.389

Rademaker, R. L., Chunharas, C., \& Serences, J. T. (2019). Coexisting representations of sensory and mnemonic information in human visual cortex. Nature Neuroscience, 22, 1336-1344. https://doi.org/10.1101/339200

Riggall, A. C., \& Postle, B. R. (2012). The relationship between working memory storage and elevated activity as measured with functional magnetic resonance imaging. Journal of Neuroscience, 32(38), 12990-12998. https://doi.org/10.1523/JNEUROSCI.1892-12.2012

Rose, N. S., LaRocque, J. J., Riggall, A. C., Gosseries, O., Starrett, M. J., Meyering, E. E., \& Postle, B. R. (2016). Reactivation of latent working memories with transcranial magnetic stimulation. Science, 354(6316), 1136-1139. https://doi.org/10.1126/science.aah7011

Schneider, D., Barth, A., \& Wascher, E. (2017). On the contribution of motor planning to the retroactive cuing benefit in working memory: Evidence by mu and beta oscillatory activity in the EEG. Neurolmage, 162(April), 73-85. https://doi.org/10.1016/j.neuroimage.2017.08.057

Serences, J. T. (2016). Neural mechanisms of information storage in visual short-term memory. Vision Research, 128, 53-67. https://doi.org/10.1016/j.visres.2016.09.010

Serences, J. T., Ester, E. F., Vogel, E. K., \& Awh, E. (2009). Stimulus-specific delay activity in human primary visual cortex. Psychological Science, 20(2), 207-214. https://doi.org/10.1111/j.14679280.2009.02276.x

Sereno, M., Dale, A., Reppas, J., Kwong, K., Belliveau, J., Brady, T., ... Tootell, R. (1995). Borders of multiple visual areas in humans revealed by functional magnetic resonance imaging. Science, 268(5212), 889-893. https://doi.org/10.1126/science.7754376

Smith, S. M. (2002). Fast robust automated brain extraction. Human Brain Mapping, 17(3), 143-155. https://doi.org/10.1002/hbm.10062

Souza, A. S., \& Oberauer, K. (2016). In search of the focus of attention in working memory: 13 years of the retro-cue effect. Attention, Perception, and Psychophysics, 78(7), 1839-1860. https://doi.org/10.3758/s13414-016-1108-5

Sprague, T. C., Boynton, G. M., \& Serences, J. T. (2019). The importance of considering model choices when interpreting results in computational neuroimaging. ENeuro, 6(6). https://doi.org/10.1523/ENEURO.0196-19.2019

Sprague, T. C., Ester, E. F., \& Serences, J. T. (2016). Restoring Latent Visual Working Memory Representations in Human Cortex. Neuron, 91(3), 694-707. https://doi.org/10.1016/j.neuron.2016.07.006

Sreenivasan, K. K., Curtis, C. E., \& D'Esposito, M. (2014). Revisiting the role of persistent neural activity during working memory. Trends in Cognitive Sciences, 18(2), 82-89.

https://doi.org/10.1016/j.tics.2013.12.001 
Supèr, H., Spekreijse, H., \& Lamme, V. A. F. (2001). A neural correlate of working memory in the monkey primary visual cortex. Science, 293(5527), 120-124. https://doi.org/10.1126/science.1060496

Swisher, J. D., Halko, M. A., Merabet, L. B., McMains, S. A., \& Somers, D. C. (2007). Visual Topography of Human Intraparietal Sulcus. Journal of Neuroscience, 27(20), 5326-5337. https://doi.org/10.1523/JNEUROSCI.0991-07.2007

van Ede, F., Chekroud, S. R., Stokes, M. G., \& Nobre, A. C. (2019). Concurrent visual and motor selection during visual working memory guided action. Nature Neuroscience, 22(3), 477-483. https://doi.org/10.1038/s41593-018-0335-6

Van Kerkoerle, T., Self, M. W., \& Roelfsema, P. R. (2017). Layer-specificity in the effects of attention and working memory on activity in primary visual cortex. Nature Communications, 8(1), 1-14. https://doi.org/10.1038/ncomms13804

Vaziri-Pashkam, M., \& Xu, Y. (2017). Goal-Directed Visual Processing Differentially Impacts Human Ventral and Dorsal Visual Representations. The Journal of Neuroscience, 37(36), 8767-8782. https://doi.org/10.1523/JNEUROSCl.3392-16.2017

Wandell, B. A., Dumoulin, S. O., \& Brewer, A. A. (2007). Visual field maps in human cortex. Neuron, 56(2), 366-383. https://doi.org/10.1016/j.neuron.2007.10.012

Winawer, J., \& Witthoft, N. (2015). Human V4 and ventral occipital retinotopic maps. Visual Neuroscience, 32, E020. https://doi.org/10.1017/S0952523815000176

Wolff, M. J., Jochim, J., Akyürek, E. G., \& Stokes, M. G. (2017). Dynamic hidden states underlying working-memory-guided behavior. Nature Neuroscience, 20(6), 864-871. https://doi.org/10.1038/nn.4546

Woolrich, M. W., Ripley, B. D., Brady, M., \& Smith, S. M. (2001). Temporal autocorrelation in univariate linear modeling of FMRI data. Neurolmage, 14(6), 1370-1386. https://doi.org/10.1006/nimg.2001.0931

Xing, Y., Ledgeway, T., McGraw, P. V., \& Schluppeck, D. (2013). Decoding working memory of stimulus contrast in early visual cortex. Journal of Neuroscience, 33(25), 10301-10311. https://doi.org/10.1523/JNEUROSCI.3754-12.2013

$\mathrm{Xu}$, Y. (2018, March 1). Sensory Cortex Is Nonessential in Working Memory Storage. Trends in Cognitive Sciences, Vol. 22, pp. 192-193. https://doi.org/10.1016/j.tics.2017.12.008

$\mathrm{Xu}, \mathrm{Y}$. (2020). Revisit once more the sensory storage account of visual working memory. Visual Cognition, 28(5-8), 433-446. https://doi.org/10.1080/13506285.2020.1818659 